COMPILATION OF CURRENT HIGH ENERGY PHYSICS EXPERIMENTS

\author{
R.L. Kelly, F.E. Armstrong, C.P. Horne, \\ M.S. Hutchinson, D.R. Richards, A. Rittenberg, \\ A.H. Rosenfeld, T.G. Trippe, G.P. Yost \\ Particle Data Group \\ Lawrence Berkeley Laboratory \\ University of California \\ Berkeley, CA 94720 USA \\ L. Addis, G.B. Chadwick, G.M. Row \\ Stanford Linear Accelerator Center \\ Stanford, CA 94305 USA \\ G.C. Fox, P.R. Stevens \\ California Institute of Technology \\ Pasadena, CA 91125 USA \\ C.E.W. Ward \\ Argonne National Laboratory \\ Argonne, Illinois 60439 USA \\ R.H. Phillips \\ Brookhaven National Laboratory \\ Uoton, New York 11973 USA \\ P. Weilhammer \\ CERN \\ CH-1211 Geneve 23, Switzerland \\ P. Joos \\ $D E S Y$ \\ Notkestieg 1 \\ D.2 Hamburg-52, Germany \\ T.H. Groves \\ Fermilab \\ P.O. Box 500 \\ Batavia, Illinois 60510 USA \\ G.T.J. Arnison \\ Rutherford High Energy Laboratory \\ Chilton, Didcot, Oxon. OX11OQX, England
}

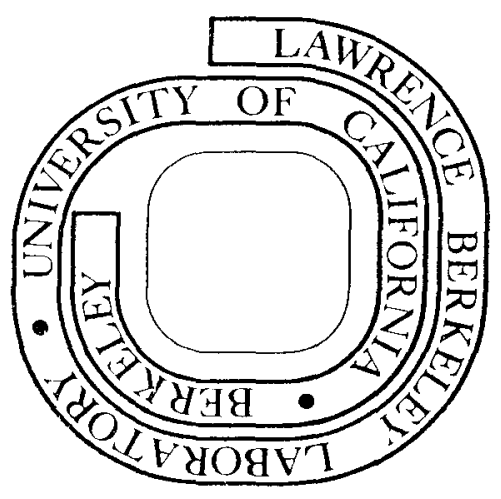

July 1976

Prepared for the U.S. Energy Research and Development Administration under Contract W-7405-ENG-48 


\section{Compilation of Current High Energy Physics Experiments}

R.L. Kelly, F.E. Armstrong, C.P. Horne, M.S. Hutchinson, D.R. Richards, A. Rittenberg, A.H. Rosenfeld, T.G. Trippe, G.P. Yost Lawrence Berkeley Laboratory

University of California

Berkeley, California 94720 USA

L. Addis, G.B. Chadwick, G.M. Row

Stanford Linear Accelerator Center

Stanford, California 94305 USA

G.C. Fox, P.R. Stevens

California Institute of Technology

Pasadena, California 91125 USA

C.E.W. Ward

Argonne National Laboratory

Argonne, Illinois 60439 USA

R.H. Phillips

Brookhaven National Laboratory

Upton, New York 11973 USA

P. Weilhammer

CERN

$\mathrm{CH}-1211$ Genève 23, Switzerland

P. Joos

DESY

Notkestieg 1

D-2 Hamburg-52, Germany

T.H. Groves

Fermilab

P.0. Box 500

Batavia, Illinois 60510 USA

G.T.J. Arnison

Rutherford High Energy Laboratory

Chilton, Didcot, Oxon. OX11 OQX, England 


\section{Table of Contents}

Introduction . . . . . . . . . . . . . . . . . . . 1

Beam-Target-komentum Index. . . . . . . . . . . . . . . 3

Particle Index

Spokesman Index . . . . . . . . . . . . . . . . . . 10

Illustrative Key for Compilation Listing . . . . . . . . . . . 12

Appendix I - Accelerator Abbreviations . . . . . . . . . . 13

Appendix II - Detector Abbreviations . . . . . . . . . . . 13

Appendix III - Beam Momentum Designations . . . . . . . . 13

Appendix IV - Reaction Data Descriptors . . . . . . . . . . 13

Appendix V - Particle Properties Descriptors . . . . . . . . 14

Appendix VI - Institution Abbreviations . . . . . . . . . . 14

Appendix VII - Particle Abbreviations . . . . . . . . . . . 17

Complete Compilation Listing (on Microfiche) . . . Inside front cover 


\section{Introduction}

This compilation of current high energy physics experiments has been assembled in a collaborative effort by the Berkeley Particle Data Group, the SLAC Library, and the seven participating laboratories, ANL, BNL, CERN, DESY, FNAL, RHEL, and SLAC. Work on the compilation was begun early in 1975 and it is now intended to include all currently active high energy physics experiments at the participating laboratories. By "currently active" we mean experments that were approved but had not finished running by June 1976. Nearly all experiments that completed running since the summer of 1975 are also included, as well as a few older experiments.

This report includes three indices to the compilation, seven appendices with definitions of the symbols used, and a microfiche listing of the compilation itself. The first index is keyed to beam and target particles and beam momenta in order of increasing particle mass and beam momentum; the two subsequent indices are keyed to individual particles studied and to spokesman names. The appendices contain lists of symbols used for accelerators, detectors, beam momentum designations, data descriptors, particle properties, institutions, and particle names. An illustrative key precedes the appendices, showing the format of the compilation listing and indicating the appropriate appendices for the various symbols used. The compilation listing for each experiment contains, in addition to the indexed information mentioned above, a list of participants and their institutions, a list of the reactions studied, information on the running status of the experiment, and various comments on the technique and/or physics goals of the experiment.

Users of this compilation who want more information about a specific experiment would be well advised to contact the experimental spokesman directly. Although proposals are sometimes available in libraries, it is not always easy to identify all the relevant information for a particular experiment. There are often important letters, addenda, revisions, etc,. attached to a proposal after it is first submitted which may not be available with library copies. The "bibliographic comments" included in the compilation listing are intended to help the user find all relevant material. Users should also note that at two laboratories the experiment number that we list and the proposal number are not the same. The ANL proposal number corresponding to experiment number $E-x x x$ is $P-x x x$. The CERN proposal number(s) corresponding to a specific experiment consists of the numbers of all CERN documents submitted in its support, and can usually be found in the CERN approved experiment list for the appropriate accelerator.

It is planned to keep this compilation up-to-date in the future, to add more laboratories, and to issue updated versions of this report every year. This report has been distributed to libraries at universities, high energy physics laboratories, etc., and to the experimental spokesmen listed in the index. A limited number of copies are also available for other interested individuals who may request a copy by writing to:

Particle Data Group
LBL-91 Request
Building 50, Room 304
Lawrence Berkeley Laboratory
Berkeley, California 94720 USA

Any comments concerning the compilation, such as missing or incorrect entries, should be sent to Robert Kelly at the Particle Data Group. 
The information appearing in this report has been extracted from a new computerized data storage and retrieval system being developed by the Berkeley Particle Data Group, in collaboration with the California Institute of Technology and the University of Durham. In the next step of its development, this "Particle Physics Data System" will contain the same kind of information as appears in this report for all experimental particle physics documents: proposals (past as well as current), preprints, reports, theses, journal articles, etc. It is also planned that it will ultimately contain the actual physics data extracted from these documents -- both reaction data and particle properties measurements. The stored material will be searchable in response to specific requests, and will also be used to produce periodic indices such as this one, as well as various graphical "trend-of-the-data" reports. We welcome suggestions concerning the development of this system.

This project is jointly supported by the U. S. Energy Research and Development Administration, the Office of the Standard Reference Data of the National Bureau of Standards, and the National Science Foundation. 
BEAM-TARGET-MOMENTUM INDEX

\begin{tabular}{|c|c|}
\hline BEAM & AND TARGET \\
\hline GAMMA & $E-$ \\
\hline GAMMA & $p$ \\
\hline GAMMA & $p$ \\
\hline GAMMA & $P$ \\
\hline GAMMA & $p$ \\
\hline GAMMA & $p$ \\
\hline GAMMA & $P$ \\
\hline GAMMA & $P$ \\
\hline GAMMA & $P$ \\
\hline GAMMA & $p$ \\
\hline GAMMA & $P$ \\
\hline GAMMA & $p$ \\
\hline GAMMA & $p$ \\
\hline GAMMA & $P$ \\
\hline GAMMA & $P$ \\
\hline GAMMA & $P$ \\
\hline GAMMA & $p$ \\
\hline GAMMA & $P$ \\
\hline GAMMA & $p$ \\
\hline GAMMA & $p$ \\
\hline GAMMA & $\mathrm{N}$ \\
\hline GAMMA & $\mathrm{N}$ \\
\hline GAMMA & $\mathrm{N}$ \\
\hline GAMMA & $N$ \\
\hline GAMMA & $\mathrm{N}$ \\
\hline GAMMA & $\mathrm{N}$ \\
\hline GAMMA & $\mathrm{N}$ \\
\hline GAMMA & N \\
\hline GAMMA & 0 \\
\hline GAMMA & 0 \\
\hline GAMMA & $B E$ \\
\hline GAMMA & $B E$ \\
\hline GAMMA & $c$ \\
\hline GAMMA & c \\
\hline GAMMA & $c$ \\
\hline GAMMA & $A L$ \\
\hline GAMMA & cu \\
\hline GAMMA & $\mathrm{cu}$ \\
\hline GAMMA & $c u$ \\
\hline GAMMA & $A G$ \\
\hline GAMMA & $\mathrm{PB}$ \\
\hline GAMHA & PB \\
\hline GAMMA & PB \\
\hline GAMMA & NUCLEUS \\
\hline GAMMA & NUCLEUS \\
\hline GAMMA & NUCLEUS \\
\hline GAMMA & NUCLEUS \\
\hline GAMMA & NUCLEUS \\
\hline GAMMA & NUCLEUS \\
\hline GAMMA & NUCLEUS \\
\hline GAMMA & NUCLEUS \\
\hline GAMMA & NUCLEUS \\
\hline GAMMA & NUCLEUS \\
\hline GAMMA & NUCLEUS \\
\hline NUE E & E- \\
\hline NUE. E & \\
\hline NUE E & $=-$ \\
\hline NUE $P$ & b \\
\hline NUE $P$ & 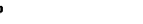 \\
\hline NUE $P$ & 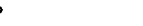 \\
\hline NUE $N$ & $\mathrm{v}$ \\
\hline NUE $\mathrm{N}$ & $\mathrm{v}$ \\
\hline ANUE & $E^{-}$ \\
\hline ANUE & $P$ \\
\hline ANUE & P \\
\hline ANUE & $\mathbf{P}$ \\
\hline ANUE & $N$ \\
\hline ANUE & $N$ \\
\hline NUMU & $E-$ \\
\hline NUMU & E- \\
\hline NUMU & $E-$ \\
\hline NUMU & E- \\
\hline NUMU & $E-$ \\
\hline NUMU & E- \\
\hline NUMU & $E-$ \\
\hline NUMU & $E-$ \\
\hline NUMU & $\mathrm{p}$ \\
\hline NUMU & $\mathrm{p}$ \\
\hline NUMU & $\mathbf{P}$ \\
\hline NUMU & $\mathrm{P}$ \\
\hline NUMU & $P$ \\
\hline NUMU & $\mathbf{P}$ \\
\hline NUMU & $\mathrm{p}$ \\
\hline NUMU & $P$ \\
\hline NUMU & $\mathrm{P}$ \\
\hline NUMU & $\mathrm{P}$ \\
\hline NUMU & $\mathbf{P}$ \\
\hline NUMU & $\mathrm{p}$ \\
\hline NUMU & $P$ \\
\hline NUMU & p \\
\hline NUNU & p \\
\hline NUMU & $\mathrm{P}$ \\
\hline NUMU & $p$ \\
\hline NUMU & P \\
\hline NUMU & p \\
\hline NUMU & P \\
\hline
\end{tabular}


BEAM-TARGET-MOMENTUM INDEX

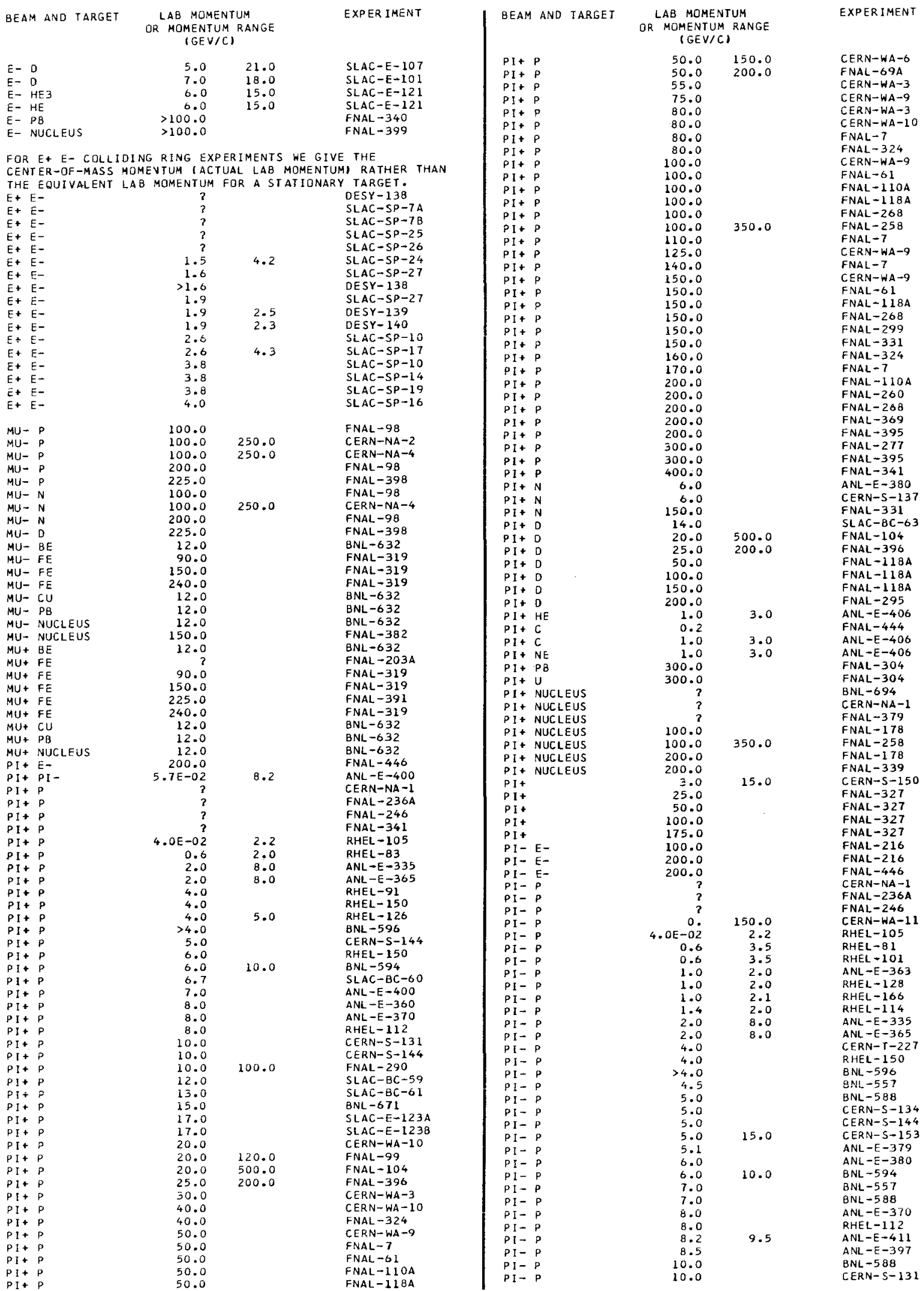


BEAM-TARGET-MOMENTUM INDEX

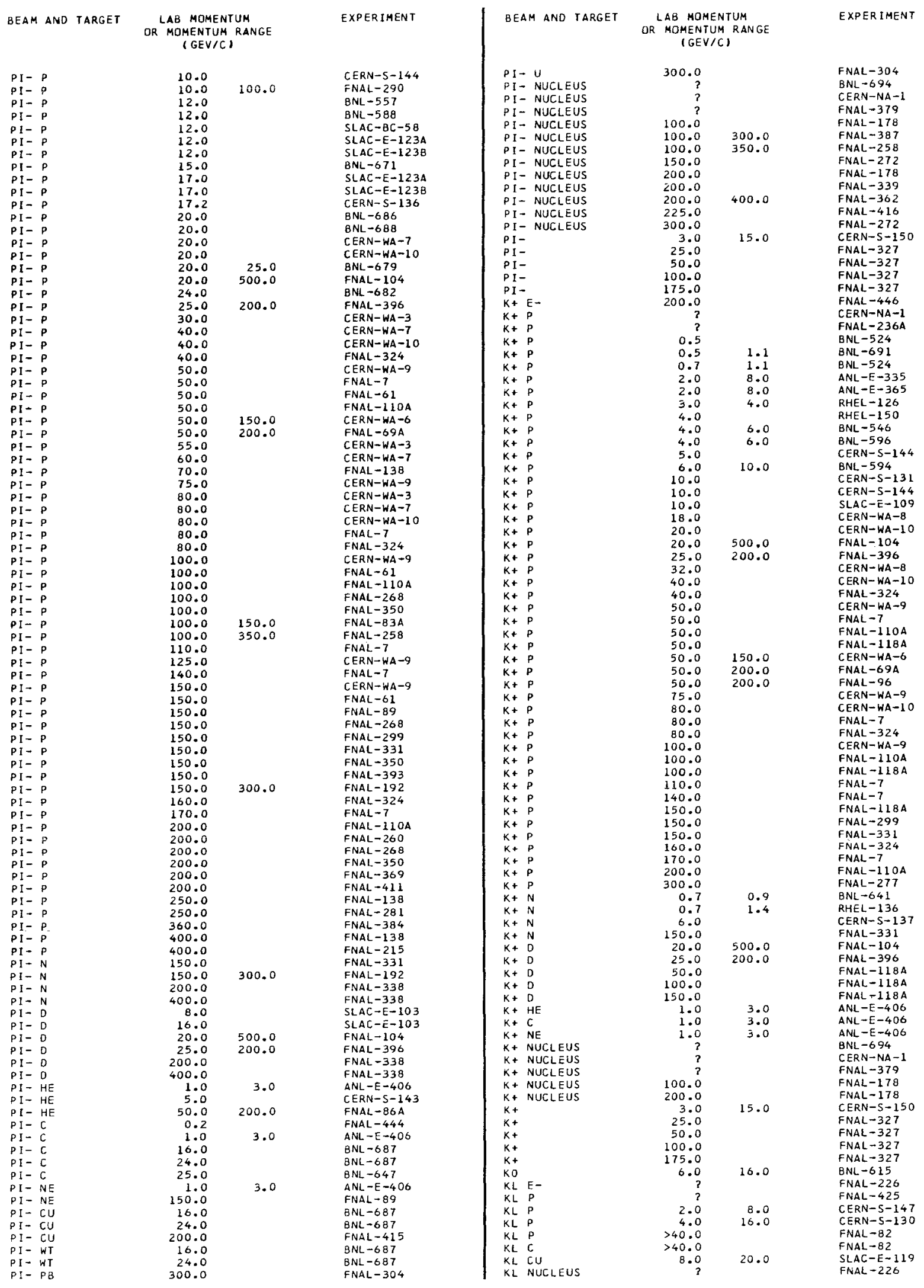


BEAM-TARGET-MOMENTUM INDEX

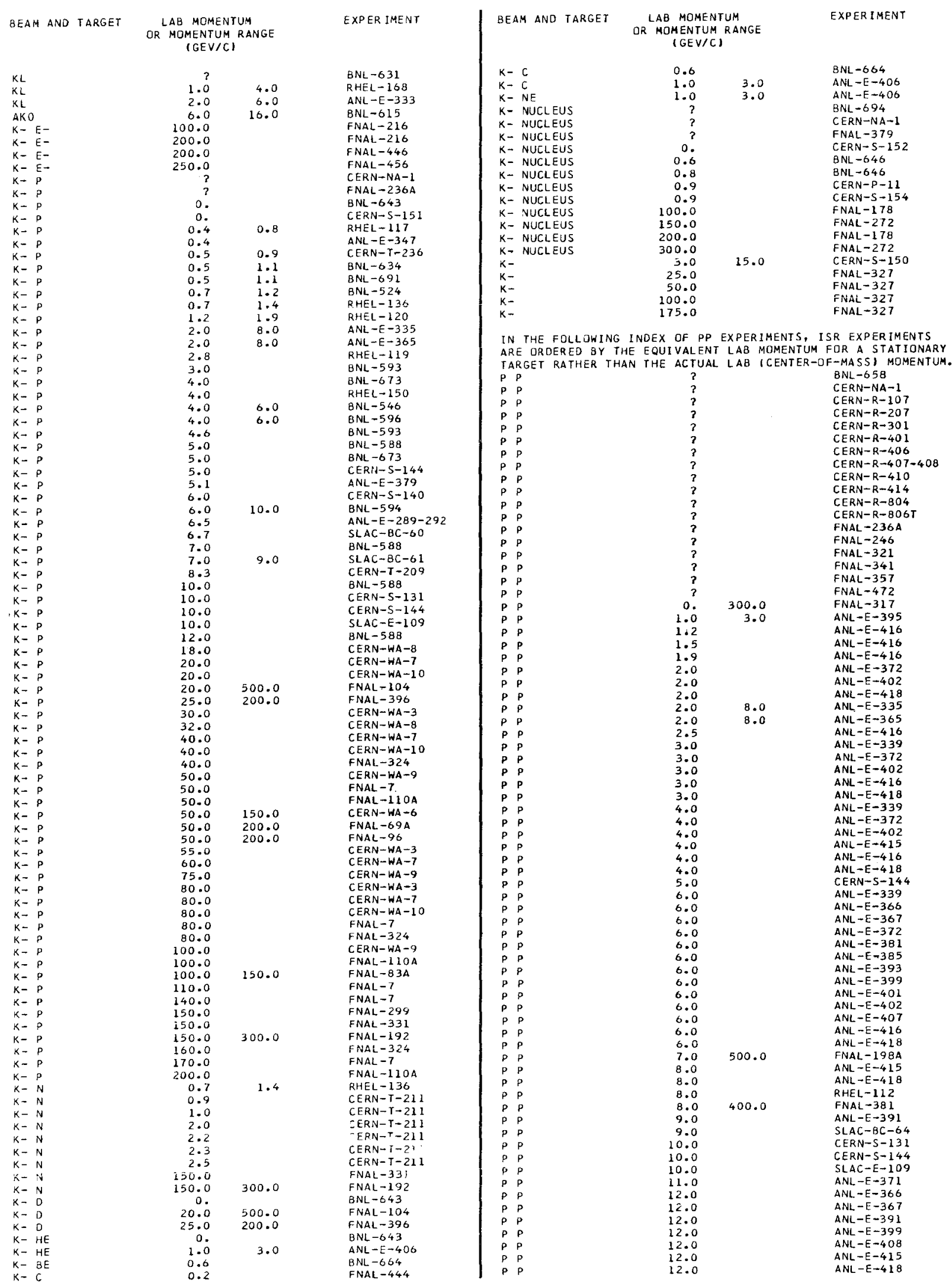


GE AM-TÁRGET-MUMENTUM INDEX

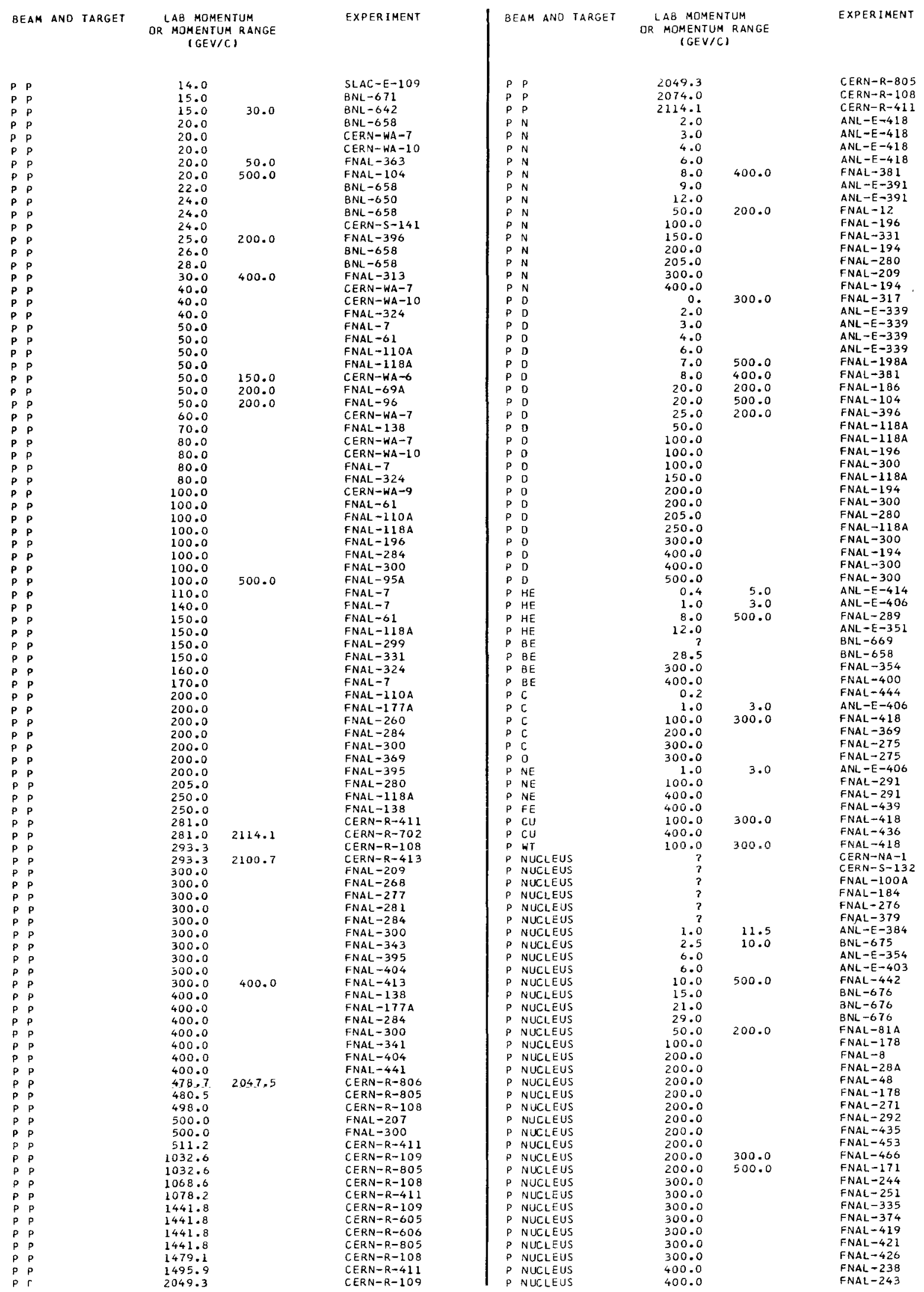


BEAM-TARGET-MOMENTUM INDEX

\begin{tabular}{|c|c|c|c|c|c|c|c|c|}
\hline \multicolumn{2}{|c|}{ BEAM AND TARGET } & $\begin{array}{r}\text { LAB MOME } \\
\text { OR MOMENTL } \\
\text { (GEV }\end{array}$ & RANGE & EXPER IMENT & BEAM AND TARGET & $\begin{array}{r}\text { LAB MO } \\
\text { OR MOMEN } \\
\text { (GE }\end{array}$ & $\begin{array}{l}\text { TUM } \\
\text { RANGE }\end{array}$ & EXPERIMENT \\
\hline $\mathrm{p}$ & NUCLEUS & 400.0 & & FNAL -245 & $A P P$ & 6.0 & 10.0 & BNL -594 \\
\hline$P_{1}$ & NUCLEUS & 400.0 & & FNAL -249 & $A P P$ & 6.3 & & BNL -654 \\
\hline p & NUCLEUS & 400.0 & & FNAL -251 & $A P P$ & 7.3 & & $C E R N \rightarrow T-237$ \\
\hline $\mathrm{p}$ & NUCLEUS & 400.0 & & FNAL -265 & $A P P$ & 8.7 & & BNL -654 \\
\hline p & NUCLEUS & 400.0 & & FNAL -279 & $A P P$ & 9.0 & & $S L A C-B C-64$ \\
\hline$P 1$ & NUCLEUS & 400.0 & & FNAL -329 & $A P P$ & 10.0 & & CERN-S-144 \\
\hline$p$ & NUCLEUS & 400.0 & & FNAL -336 & $A P P$ & 10.0 & & $5 L A C-E-109$ \\
\hline $\mathrm{P}$ & NUCLEUS & 400.0 & & FNAL -346 & $A P P$ & 10.0 & 12.0 & BNL-661 \\
\hline$p 1$ & NUCLEUS & 400.0 & & FNAL -364 & $A P P$ & 12.0 & & CERN $-T-243$ \\
\hline p & NUCLEUS & 400.0 & & FNAL-385 & $A P P$ & 15.0 & & $B N L-671$ \\
\hline$p$ & NUCLEUS & 400.0 & & FNAL -423 & $A P P$ & 20.0 & & CER N $-W A-7$ \\
\hline$p$ & NUCLEUS & 400.0 & & FNAL -428 & $A P P$ & 20.0 & & CERN-WA-10 \\
\hline$p$ & NUCLEUS & 400.0 & & FNAL -434 & AP $P$ & 20.0 & 500.0 & FNAL- 104 \\
\hline$p_{i}$ & NUCLEUS & 400.0 & & FNAL -461 & AP $P$ & 25.0 & 200.0 & FNAL -396 \\
\hline p & NUCLEUS & 400.0 & & FNAL-462 & $A P P$ & 30.0 & 60.0 & FNAL -344 \\
\hline$p$ & NUCLEUS & 400.0 & & FNAL -463 & $A P P$ & 40.0 & & CERN-WA-7 \\
\hline$p$ & NUKLEUS & 500.0 & & FNAL -48 & $A P P$ & 40.0 & & CERN-WA-10 \\
\hline$p_{1}$ & NUCLEUS & 500.0 & & FNAL -249 & $A P P$ & 40.0 & & FNAL -324 \\
\hline$p$ & NUCLEUS & 500.0 & & FNAL -271 & $A P P$ & 50.0 & & CERN-WA-9 \\
\hline$p$ & NULLEUS & 500.0 & & FNAL-288 & $A P P$ & 50.0 & & FNAL -7 \\
\hline$p$ & NUCLEUS & 500.0 & & FNAL -292 & AP $P$ & 50.0 & & FNAL-110A \\
\hline p & NUCLE US & 500.0 & & FNAL -329 & $A P P$ & 50.0 & 200.0 & FNAL $=69 A$ \\
\hline$P$ & NUCLEUS & 500.0 & & FNAL -435 & $A P P$ & 50.0 & 200.0 & FNAL -96 \\
\hline $\mathrm{p}$ & & 3.0 & 15.0 & CERN-S-150 & $A P P$ & 60.0 & & CERN-WA-7 \\
\hline$p$ & & 25.0 & & FNAL -327 & $A P P$ & 75.0 & & CERN-WA-9 \\
\hline$p$ & & 50.0 & & FNAL $=327$ & $A P P$ & 80.0 & & CERN-WA-7 \\
\hline$p$ & & 50.0 & 500.0 & FNAL -108 & $A P P$ & 80.0 & & CERN-WA-10 \\
\hline$p$ & & 100.0 & & FNAL -327 & $A P P$ & 80.0 & & FNAL -7 \\
\hline$p$ & & 175.0 & & FNAL -327 & $A P P$ & 80.0 & & FNAL -324 \\
\hline N & P & 25.0 & 200.0 & FNAL-396 & $A P P$ & 100.0 & & CERN-WA -9 \\
\hline $\mathrm{N}$ & p & 40.0 & 300.0 & FNAL -4 & $A P P$ & 100.0 & & FNAL- $110 A$ \\
\hline $\mathrm{N}$ & $\mathrm{P}$ & 40.0 & 300.0 & FNAL -248 & $A P P$ & 100.0 & & FNAL -311 \\
\hline $\mathrm{Ni}$ & p & 50.0 & 300.0 & FNAL -305 & $A P P$ & 100.0 & 150.0 & FNAL $-83 A$ \\
\hline $\mathrm{N}$ & p & 100.0 & 200.0 & FNAL -366 & $A P P$ & 110.0 & & FNAL-7 \\
\hline $\mathrm{Ni}$ & $\mathrm{N}$ & 50.0 & 300.0 & FNAL -305 & $A P P$ & 140.0 & & FNAL-7 \\
\hline N & D & 25.0 & 200.0 & FNAL- 396 & $A P \quad P$ & 150.0 & & FNAL-299 \\
\hline N & 0 & 40.0 & 300.0 & FNAL -4 & $A P P$ & 150.0 & & FNAL -311 \\
\hline $\mathrm{N}$ & D & 100.0 & 200.0 & FNAL -366 & $A P P$ & 150.0 & & FNAL -331 \\
\hline $\mathrm{N}$ & $B E$ & $>6.0$ & & $8 N L-656$ & $A P P$ & 150.0 & 300.0 & FNAL -192 \\
\hline N & $\mathrm{BE}$ & 30.0 & 300.0 & FNAL -438 & $A P P$ & 160.0 & & FNAL -324 \\
\hline$N$ & $B E$ & 200.0 & & FNAL -397 & $A P P$ & 170.0 & & FNAL -7 \\
\hline N & $c$ & 30.0 & 300.0 & FNAL -438 & $A P P$ & 200.0 & & FNAL-110A \\
\hline $\mathrm{N}$ & AL & 30.0 & 300.0 & FNAL -438 & $A P P$ & 200.0 & & FNAL-302 \\
\hline $\mathrm{N}$ & $\mathrm{FE}$ & 30.0 & 300.0 & FNAL $=438$ & $A P P$ & 200.0 & & FNAL-392 \\
\hline N & $\mathrm{CU}$ & 30.0 & 300.0 & FNAL -438 & $A P N$ & 100.0 & & FNAL -345 \\
\hline n & $C D$ & 30.0 & 300.0 & FNAL -438 & $A P N$ & 150.0 & & FNAL -331 \\
\hline $\mathrm{N}$ & WT & 30.0 & 300.0 & FNAL -438 & $A P N$ & 150.0 & 300.0 & FNAL -192 \\
\hline $\mathrm{N}$ & $P B$ & 30.0 & 300.0 & FNAL -438 & $\triangle P D$ & $?$ & & $B N L-660$ \\
\hline $\mathrm{N}$ & $u$ & 30.0 & 300.0 & FNAL -438 & $A P D$ & 0. & & BNL -643 \\
\hline $\mathrm{N} N$ & NUCLEUS & 40.0 & 300.0 & FNAL -4 & $A P D$ & 0. & 0.7 & $A N L-E-413$ \\
\hline $\mathrm{Ni}$ & NUCLEUS & 50.0 & 300.0 & FNAL -305 & $A P D$ & 2.0 & 4.0 & $B N L-625$ \\
\hline $\mathrm{N} N$ & NUCLEUS & 100.0 & 200.0 & FNAL -366 & $A P \quad D$ & 20.0 & 500.0 & FNAL -104 \\
\hline $\mathrm{N}$ & NUCLEUS & 300.0 & & FNAL- 358 & $A P D$ & 25.0 & 200.0 & FNAL-396 \\
\hline $\mathrm{n} N$ & NUCLEUS & 400.0 & & FNAL -358 & AP HE & 0. & & $B N L-643$ \\
\hline AN & $\mathrm{P}$ & 0 & 0.8 & $A N L-E-303$ & AP $\mathrm{HE}$ & 1.0 & 3.0 & $A N L-E=406$ \\
\hline AN & $P$ & $5.0 \mathrm{E}-02$ & 0.7 & $B N L-626$ & $A P C$ & 1.0 & 3.0 & ANL $-E-406$ \\
\hline$A N$ & $P$ & 50.0 & 300.0 & FNAL -305 & AP NE & $?$ & & $A N L-E-409$ \\
\hline$A N$ & $\mathrm{~N}$ & 50.0 & 300.0 & FNAL -305 & AP NE & 1.0 & 3.0 & $A N L-E-406$ \\
\hline$A N$ & NUCLEUS & 50.0 & 300.0 & FNAL-305 & AP NUCLEUS & $?$ & & $B N L-694$ \\
\hline$A P$ & $\mathrm{p}$ & $?$ & & $A N L-E-368$ & AP NUCLEUS & $?$ & & CERN-NA-1 \\
\hline AP & $p$ & $?$ & & $A N L-E-409$ & AP NUCLEUS & $?$ & & FNAL -379 \\
\hline$A P$ & p & $?$ & & $B N L-660$ & AP NUCLEUS & 10.0 & 12.0 & $B N L-661$ \\
\hline$A P$ & $p$ & $?$ & & CERN-NA-I & AP NUCLEUS & 150.0 & & FNAL -272 \\
\hline$A P$ & $p$ & $?$ & & FNAL $-236 A$ & AP NUCLEUS & 300.0 & & FNAL $=272$ \\
\hline$A P$ & $p$ & 0. & & $B N L-643$ & $A P$ & 3.0 & 15.0 & CERN-S -150 \\
\hline$A P$ & $p$ & 0 . & & CERN-S-135 & $A P$ & 25.0 & & FNAL -327 \\
\hline$A P$ & $p$ & 0. & & CERN-S-142 & $A P$ & $50: 0$ & & FNAK -327 \\
\hline$A P$ & $p$ & 0. & & CERN-S-151 & $A P$ & 100.0 & & FNAL -327 \\
\hline$A P$ & $p$ & 0. & 0.7 & $A N L-E-413$ & $A P$ & 175.0 & & FNAL -327 \\
\hline$A P$ & $p$ & 0. & 0.8 & $A N L-E-303$ & LAMBOA P & 60.0 & 130.0 & FNAL-8 \\
\hline$A P$ & - $p$ & 0.3 & 1.0 & $B N L-634$ & LAMBOA P & 75.0 & 150.0 & FNAL-97 \\
\hline$A P$ & $P$ & 0.3 & 1.0 & $B N L-666$ & LAMBDA & 6.0 & 15.0 & $8 N L-597$ \\
\hline$A P$ & $p$ & 0.4 & & BNL. -662 & LAMBDA & 150.0 & 210.0 & FNAL -440 \\
\hline$A P$ & $p$ & 0.4 & 0.9 & CERN-S-149 & ALAMBDA $P$ & 60.0 & 130.0 & FNAL- 8 \\
\hline$A P$ & $p$ & 0.5 & & $B N L=662$ & SIGMAt & 100.0 & & CERN-WA-2 \\
\hline$A P$ & - $P$ & 0.6 & & $B N L-662$ & SIGMA-P & 75.0 & 150.0 & FNAL -97 \\
\hline$\Delta P$ & $p$ & 1.2 & & $A N L-E-382$ & SI GMA- & $?$ & & $B N L-618$ \\
\hline$A P$ & $\mathrm{p}$ & 1.2 & 3.0 & $B N L-644$ & SI GMA- & 100.0 & & CERN $-W A-2$ \\
\hline $\mathrm{AP}$ & $p$ & 2.0 & & RHEL-115 & $X I-P$ & 75.0 & 150.0 & FNAL $=97$ \\
\hline$A P$ & $p$ & 2.0 & 8.0 & $A N L-E-365$ & $x_{1}-$ & $?$ & & $B N L-618$ \\
\hline$A P$ & $p$ & 2.6 & & $A N L-E-383$ & $\hat{x}_{I}-$ & 100.0 & & CERN-WA-2 \\
\hline$A P$ & $p$ & 3.9 & & BNL -654 & OMEGA-P & 75.0 & 150.0 & FNAL -97 \\
\hline$A P$ & $p$ & 4.0 & 10.0 & BNL-596 & HADRON $P$ & 3 & & CERN-NA-3 \\
\hline$A P$ & $p$ & 5.0 & & CERN-S-144 & HADRON $P$ & ? & & CERN-NA-5 \\
\hline$A P$ & $p$ & 5.7 & & $A N L-E-383$ & NEUTRAL NUCLEUS & $?$ & & FNAL -386 \\
\hline$A P$ & $p$ & 6.0 & & $8 N L-601$ & & & & \\
\hline
\end{tabular}


PARTICLE INDEX

\begin{tabular}{|c|c|c|c|}
\hline PARTICLE & EXPERIMENTISI & PARTICLE & EXPERIMENT(S) \\
\hline Mu- & CERN-S-97 & SIGMA- & ANL-E-347: BNL-618; CERN-WA-2 \\
\hline Mut & CERN-5-97 & SIG(1385P13)+ & $A N L-E-289-292$ \\
\hline HVY-LEPTON+ & FNAL -192 & SIG(1385P13)- & $A N L-E-289-292$ \\
\hline HVY-LEPTON- & FNAL -192 & $S I G(1670 B)+$ & $A N L-E-289-292$ \\
\hline HVY-LEPTONO & FNAL -192 & $\operatorname{SIG}(16708) 0$ & $A N L-E-289-292$ \\
\hline HVY-LEPTON & 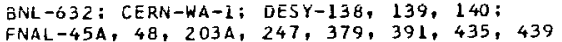 & $51 G(16708)-$ & $\begin{array}{l}A N L-E-289-292 \\
\text { AN: }-E-289-292\end{array}$ \\
\hline AHVY-LEPTON & $\begin{array}{l}\text { FNAL-45A, 48, 203A, 247, 379, 391, 435, } 439 \\
\text { FNAL-172, } 180\end{array}$ & $\begin{array}{l}\text { SIG } \\
\text { SIG(1915B) }\end{array}$ & $\begin{array}{l}A N L-E-289-292 \\
\text { ANL }-E+289-292\end{array}$ \\
\hline $\mathrm{PI+}$ & FNAL -446 & $516(1915 B)-$ & $A N L-E-289-292$ \\
\hline PIO & FNAL $-171,265$ & $516(22508)+$ & $A N L-E-289-292$ \\
\hline PI- & FNAL -446 & SIG $(22508) 0$ & $A N L-E-289-292$ \\
\hline EPSILON $(700)$ & ANL $-E-370, E-400$ & $5 \mathrm{IG}(22508)-$ & $A N L-E-289-292$ \\
\hline RHOO & $D E S Y-115 ; \quad S L A C-E-123 A$ & $x+0$ & FNAL -8 \\
\hline RHO- & FNAL -272 & $x_{I}-$ & $B N L-618 ;$ CERN-WA-2 \\
\hline OMEGA & $D E S Y-115$ & $X I(1530 P 13) 0$ & ANL-E-289-292: RHEL-119 \\
\hline ETAPR IME & ANL $-E-289-292: B N L-593:$ RHEL-128 & $X I(1530 P 13)-$ & ANL-E-289-292: BNL-673; RHEL-119 \\
\hline DELTA $(970)+$ & $A N L-E-289-292, E-382, E-397$ & $x I 1163010$ & ANL $-E-289-292:$ RHEL -119 \\
\hline DELTA $(970)$ - & $A N L-E-289-292, E-382, E-397 ; B N L-557,593$ & $x I 116301-$ & ANL $-E-289-292 ;$ RHEL-119 \\
\hline $\mathrm{H}(990)$ & $A N L-E-397$. & $x 11182010$ & ANL-E-289-292: RHEL-119 \\
\hline $\begin{array}{l}\text { S*19931 } \\
\text { PHI }\end{array}$ & $A N L-E-370 ; R H E L-128$ & $x \pm(1820)-$ & ANL-E-289-292; BNL-673; RHEL-119 \\
\hline $\begin{array}{l}\text { PHI } \\
\text { EIA(1080) }\end{array}$ & DESY -115 & $x I 1194010$ & ANL-E-289-292: RHEL-119 \\
\hline ETA(1080) & $A N L-E-368$ & $X I(1940)-$ & ANL-E-289-292; BNL-673; RHEL-119 \\
\hline Al11100)0 & $A N L-E-397$ & $X I(203010$ & $A N L-E-289-292$ \\
\hline & $A N L-E-289-292$ & $x I(2030)-$ & $A N L-E-289-292$ \\
\hline$D(1285)$ & $A N L-E-368, E-382, E-397$ & $x I 1225010$ & ANL-E-289-292 \\
\hline A2(1310)+ & RHEL $\rightarrow 91$ & $X I(2250)-$ & $A N L-E-289-292$ \\
\hline A2(1310)- & FNAL -272 & $X I 1250010$ & $A N L-E-289-292$ \\
\hline E(1420) & $A N L-E-368, E-382$ & $x+12500)-$ & $A N L-E-289-292$ \\
\hline FPRIME & $A N L-E-289-292$ & XI (UNSPEC) & CERN-T -209 \\
\hline F1(1540)0 & $B N L-593 ;$ CERN-HA-8 & $X I *(U N S P E C)$ & $B N L-593$ \\
\hline$A 3(1640)-$ & $B N L-557$ & OMEGA- & CERN-T -209 \\
\hline G(1680)- & $B N L-557$ & HE3 & SLAC-E-121 \\
\hline $5119301-$ & $A N L-E-413$ & HE & $S L A C-E-121$ \\
\hline $\begin{array}{l}\text { J/PSI } 1310010 \\
\text { PSI } 1370010\end{array}$ & ANL-E-411: OESY-140: FNAL-391, 444 & $\begin{array}{l}\text { HNUCLEUS } \\
\text { OUARK }(2(3)\end{array}$ & $\begin{array}{l}\text { CERN-S-152, S- } 154 \\
\text { FNAL }-89\end{array}$ \\
\hline $\begin{array}{l}\mathrm{PSI}(3700) 0 \\
\mathrm{~K}+\end{array}$ & $\begin{array}{l}\text { DESY-138, 140; FNAL-391, 444; SLAC-SP-27 } \\
\text { FNAL }-446\end{array}$ & QUARK $(1,3)$ & FNAL-89 \\
\hline KS & $A N L-E-363 ; 8 N L-615$ & QUARK & FNAL $-45 \mathrm{~A}$ \\
\hline $\mathrm{KL}$ & $\begin{array}{l}A N L-E-333, E-363 ; B N L-631 ; F N A L-82,425 ; \\
R H E L-168\end{array}$ & Wo & $\begin{array}{l}\text { FNAL }-21 A, 45 A, 48,53 A, 192,288,435 \\
\text { CERN-R-804: FNAL-288 }\end{array}$ \\
\hline k- & FNAL $-446,456$ & $H-$ & FNAL $-172,180,192,288,411$ \\
\hline$K *(892)-$ & FNAL -272 & $w$ & CERN-WA-1: FNAL-310, 379 \\
\hline$K *(1420)+$ & $8 N L-546$ & $5+$ & FNAL $-53 A, 180$ \\
\hline$k \neq(1420)-$ & $B N L-546: F N A L-272$ & $5-$ & FNAL -180 \\
\hline KN(1700)+ & CERN-S-131 & CHARM & BNL-654, 658, 669, 679, 682, 686, 688, 694: \\
\hline KN(1700)- & CERN-S-131 & & $\begin{array}{l}\text { CERN-R-605, R-702, T-243, T-245: DESY-138, } \\
139 \text { : FNAL-151A, } 247,346,357,364,369,\end{array}$ \\
\hline $\begin{array}{l}\text { L }(1770)- \\
K \$(U N S P E C)-\end{array}$ & $\begin{array}{l}A N L-E-289-292 \\
A N L-E-289-292\end{array}$ & & \\
\hline & $B N L-643$ & & $416,419,428,472:$ SLAC-E-123B \\
\hline N(14708)+ & $A N L-E-339$ & TACHYON & FNAL -202 \\
\hline$N(15200)+$ & $A N L-E-339$ & MONOPOLE & CERN-R-109, R-301: FNAL-3, 25A, 45A, 76, \\
\hline N1 1520810 & $A N L-E-339$ & & 202 \\
\hline N(1700B)t & $A N L-E-339$ & UNSPEC & CERN-R-406: FNAL-45A, 171, 184, 238, 243, \\
\hline N1 1700810 & ANL $-E-339$ & & 421,423 \\
\hline$D E L(1232 P 33)++$ & $A N L \rightarrow E-339$ & CHARGEOH & FNAL- $276 ;$ RHEL -144 \\
\hline DEL(19508)t+ & $A N L-E-339$ & CHARGED- & FNAL-276 \\
\hline LAMBOA & BNL-597, 618: FNAL -440 & NEUTRAL & FNAL $-330,366$ \\
\hline SIGMA+ & CERN-WA-2 & & \\
\hline
\end{tabular}


SPOKESMAN INOEX

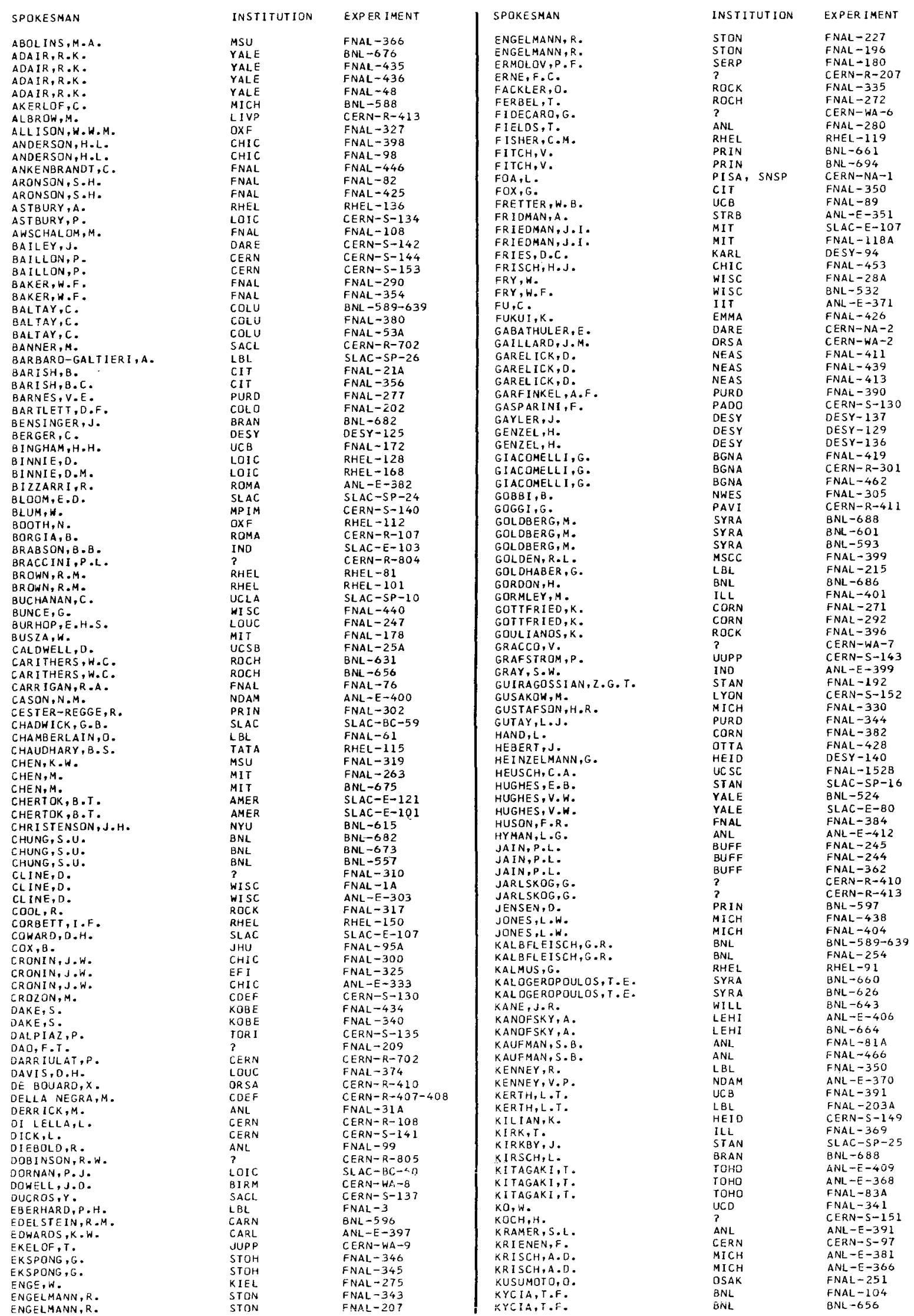


SPOKESMAN INDEX

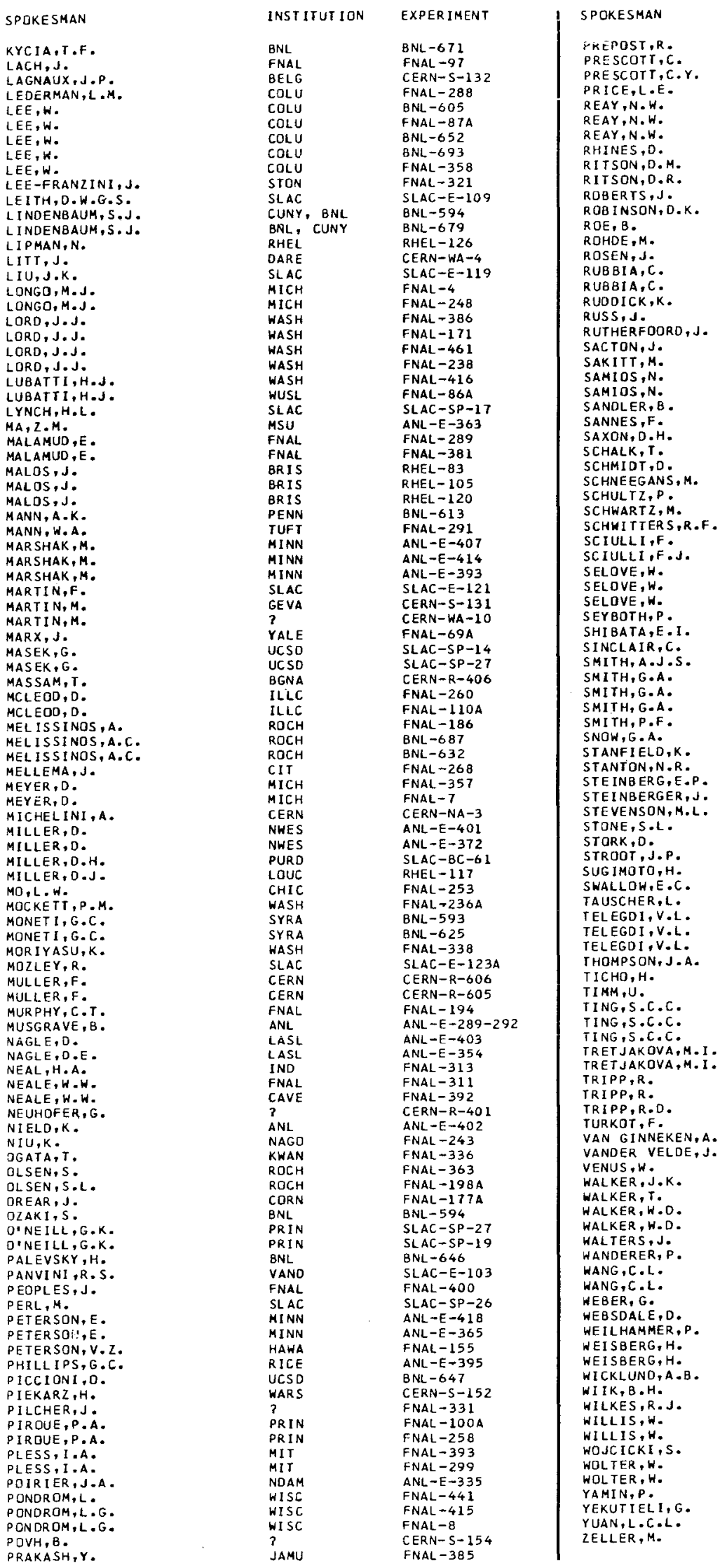

INSTITUTION EXPERIMENT

WISC

$\begin{array}{ll}\text { SLAC } & \text { SLAC-E-122 } \\ \text { SLAC } & \text { SLAC-E-95 } \\ \text { COLU } & \text { ANL-E-415 } \\ \text { OSU } & \text { FNAL-12 } \\ \text { OSU } & \text { FNAL-366 } \\ \text { OSU } & \text { ANL-E-380 } \\ \text { ILL } & \text { ANL-E-360 } \\ \text { STAN } & \text { FNAL-96 } \\ \text { SLAC } & \text { SLAC-E-112 } \\ \text { RICE } & \text { ANL-E-408 } \\ \text { CASE } & \text { ANL-E-367 } \\ \text { MICH } & \text { FNAL-45A } \\ \text { ? } & \text { DESY }-115 \\ \text { NWES } & \text { FNAL-397 } \\ \text { ? } & \text { CERN-NA-4 } \\ \text { HARV } & \text { BNL-613 } \\ \text { MINN } & \text { ANL-E-411 } \\ \text { CARN } & \text { BNL-642 } \\ \text { TUFT } & \text { SLAC-E-108 } \\ \text { BRUX } & \text { FNAL-364 } \\ \text { BNL } & \text { BNL-641 }\end{array}$

FNAL -364

BNL-629

BNL-427

ANL $-E-385$

FNAL -418

RHEL -166

$S L A C-E-123$

DESY -126
CERN-S 135

CERN $-S-135$
ANL $\rightarrow E-413$

$B N L-614$

$S L A C=S P-17$

FNAL -320

FNAL -356

FNAL -246

$B N L-557$

FNAL -395
CERN $-N A-5$

ANL $-E=379$

SLAC-E-122

ANL-E-289-292

FNAL -281

SLAC $\rightarrow B C-64$

FNAL $-151 \mathrm{~A}$

FNAL -1511 A

FNAL -472

ANL $=E-380$

CERN-WA-1

FNAL -388

SLAC-E- 103

FNAL- 216

CERN-5-132

FNAL -423

ANL-E-347

CERN-S-151

FNAL -425

FNAL -82

FNAL -226

$\mathrm{BNL}-618$

SLAC-BC-58

DESY -138

$B N L-669$

CERN-R-804

FNAL -329
FNAL -463

BNL-666

BNL-691

BNL -634

FNAL -442

FNAL -276

FNAL -138
ANL $-E-383$

FNL $-E-38$

RHEL -114

SLAC-BC-63

SLAC-BC- 63

FNAL -184

BNL-664

ANL $-E-406$

DE SY-114

CERN-S-134

CERN-HA-3

BNL-650

FNAL -324

ANL-E $=339$
$O E S Y=139$

FNAL -387

CERN-R-B06

FNAL -379

FNAL -249

FNAL -339

$B N L-644$

FNAL -295

CERN-R-107
BNL-662 
FNAL-8 (JUN 1970); APPROVED AUG 1970; STARTED; COMPLETED 22 MAR 19T6. EXPERIMENT NUMBER AND STATUS. PROPOSAL DATE IN PARENTHESES. EXPERIMENTS IN A NEUTRAL HYPERON BEAM —PROPOSAL TITLE ANDIOR BRIEF DESCRIPTION (DESCRIPTION ENCLOSED IN BRACKETS).

WISC - R. R.MARCH, L.G.PONDROM(SPOKESMAN)

MICH - - O.E. OVERSÉTH

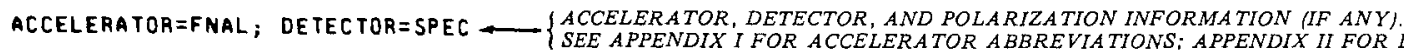

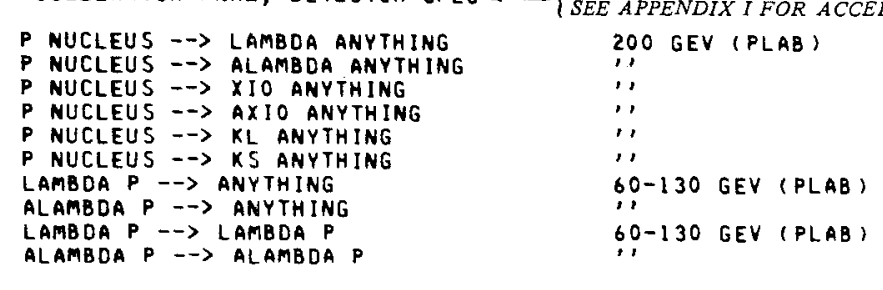

200 GEV (PLAB)
$\because:$,
$\because,:$
$:$,
$: \because-130$ GEV (PLAB)
$: \because 0-130$ GEV (PLAB)

$X I 0$

$\mathrm{PW}$

\EXPERIMENTAL COMMENT> NUCLEAR PAOOUCTION MEASUREMENTS ARE ZERO DEGREE YIELDS FOR HYPERON

BEAM; APPROVED FOR 400 HOURS; RAN 2350 HOURS.

FNAL-12 ( 12 JUN 1970); APPROVED AUG 19T0; STARTED; COMPLETED 2 DEC 1974.

A STUOY OF NEUTRON-PROTON CHARGE-EXCHANGE SCATTERING IN THE MOMENTUM RANGE 50-200 GEV/C

OSU -- N.W.REAYY SPOKESMAN), K.REIBEL, T.A.ROMANOWSKI, N.R.STANTON, J.S.FITCH, C.J.RUSH) INSTITUTIONS AND PARTICIPANTS. SEE APPENDIX VI FOR

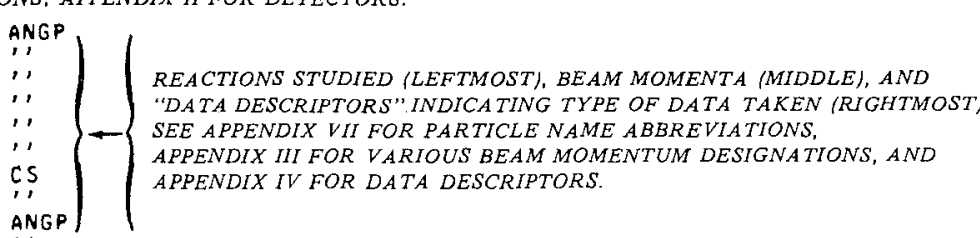

CARL - M.A.ABOL INS, M.T.LIN, G.A.SMITH

ACCELERA TOR=FNAL; DETECTOR=SPEC

$P N \rightarrow N P$

$50-200 \mathrm{GEV}$ (PLAB)

ANGP

SDATA COMMENTS SMALL ANGLE CEX, $-T=.002-1.0$ GEV 2
SEXPERIMENTAL COMMENT) APPROVED FOR 600 HQURS, RAN FOR 1300 HOURS

FNAL-21A (10 JUN 1970); APPROVED OCT 1970; STARTED; COMPLETEO 2 NOV 1975

NEUTRINO PHYSICS AT YERY HIGH ENERGIES COLLABORATION NAME (IF ANY) APPEARS HERE.

CIT -- F.SCIULLI, B.BARISHISPOKESMAN), W.FORD, P.ODOONE, C.PECK

FNAL -- A.MASCHKE

ACCELERA TOR=FNAL; DETECTOR=COMB

NUMU NUCLEUS $\rightarrow \rightarrow$ MU- ANYTHING

NUMU NUCLEU $5 \rightarrow$ NUCLEUS MU- MU+ NUMU 300 GEV (PLAB)

CS, ANGP

w+

EX,OEC — INDIVIDUAL PARTICLES (LEFT) AND PARTICLE PROPERTIES (RIGHT)

EXPERIMENTAL COMMENT> MONOCHROMATIC NEUTRINO BEAM; APPROVED FOR 1200 HOUAS; RAN FOR 2450

HOURS.
CBIBLIOGRAPHIC COMMENT> DOCUMENT HAS ONE ADDENDUM AND IS NUMBERED FNAL-2I IN SLAC

COLLECTION 
Appendix I - Accelerator Abbreviations

The abbreviations used for high energy physics facilities are listed below. For The to the institution abbreviation.

\begin{tabular}{ll} 
ANL & ARGONNE (ZCS) PROTON SYNCH. (12.7 GEV) \\
BNL & BROOKHAVEN (AGS) PROTON SYNCH. (33 GEV) \\
CERN & CERN (CPS) PROTON SYNCH. (28 GEV) \\
CERN-ISR & CERN (ISR) PROTON-PROTON ISR (11-31 GEV) \\
CERN-SPS & CERN PROTON SYNCH (400 GEV) \\
COSM & COSMIC RAYS \\
DESY & HAMBURG DEUTCHES ELECTRON SYNCH. (7.5 GEV) \\
DESY-DORIS & HAMBURG (DORIS) ELECTRON-POSITRON RING (3 GEV) \\
FNAL & FNAL BATAVIA PROTON SYNC. (500 GEV) \\
RHEL & RUTHERFORD (NIMROD) PROTON SYNCH. (8 GEV) \\
SLAC & STANFORD ELECTRON LINEAR ACCEL. (22 GEV) \\
SLAC-SPEAR & STANFORD (SPEAR) ELECTRON-POSITRON RING (4.2 GEV) \\
\hline
\end{tabular}

Appendix II - Detector Abbreviations

The abbreviations used for non-bubble-chamber detectors are as follows:

CALO Colorimeler

COMB Combinations of different types of detectors. Can include a hybrid system involving a bubble chamber, if the bubble chamber is a minor part of the system.

EMUL Emulsion. Also used for detectors like plastic where tracks are "frozen" in a solid medium.

OTHER Rare non-electronic detectors.

DAS Double Arm Spectrometer

WAS Wide Angle Spectrometer

SPEC General spectrometer system not fitting one of the above or where specific type not given. For other electronic detector systems, not including magnets for momentum

CNTR Counters (no chambers)

WIRE Wire chambers (proportional wire chambers, drift chambers).

SPRK Spark chamber of unspecified type.

DASP DPecific devices:
DESY Double Arm Spectrometer System

EMS Argonne Effective Mass Spectrometer

LASS SLAC Large Aperture Solenoid Spectrometer

OMEGA CERN OMEGA Spectrometer

PLUTO DESY Superconducting Solenoid Spectrometer

RMS Rutherford Magnetic Spectrometer Facility

SFM CERN-ISR Split Field Magnet

For bubble chambers we use a hyphenated construction, such as hydr-1SF-AYB, where the frst or heavy HBC, DBC, HEBC, or HLBC indicates indicates the size or name, and the third element, HYP, RAP, or TST, is included if the device is hybrid, rapid cycling, or track sensitive.
Appendix III - Beam Momentum Designations

The beam "momentum" designation given in parentheses following the numerical value and units can be one of the following:

PLAB beam momentum in the lab frame.

TLAB beam kinetic energy in the lab frame.

ECM total energy in the CM frame.

$5 \quad$ lotal $C M$ energy squared.

For colliding beam experiments the momentum of the second beam is given indented below that of the first. Alternately, a single line with the tot For energy or equivalent lab beam momentum may be given.

or electroproduction or other reactions involving a virtual photon, the second and third lines indented below the beam momentum specify the the following designations:

W mass of the target-virtual photon system.

W2 square of $\mathrm{W}$.

value of the mass squared of the virtual photon = value of the squared 4-momentum transfer to the electron. electron in the lab frame.

\section{Appendix IV - Reaction Data Descriptors}

The data descriptors refer to the nature of the data taken in an experiment. Any of the variables below can also be understood to refer to functions (including averages or other moments, but not derivatives or integrals) of that variable, unless such functions involve other variables from the list. Por dala which represent a un cuction of

\section{GENERAL}

CS Cross section, cross section ratio, and cross section upper limit. Can also be listed for very rare reactions whose existence is being established, even though the number of events is not converted to a cross section. Does not include parametriza.ted as a function of energy.

ANGP Production angular distribution, i.e., of one or more of the outgoing particles relative to one of the incident particles. Includes $d \sigma / d \Omega$, $\mathrm{d} \sigma / \mathrm{dt}, \mathrm{d} \sigma / \mathrm{dt}, \mathrm{d} \sigma / \mathrm{dQ}^{2}$, etc. Also the equivalent, expressed as moments or polynomial expansion coefficients. Also invariant cross section as a function of production angle or $t$. By convention, does
not include rapidity or its approximation, $y \propto-\ell \mathrm{n} \tan \theta / 2$ (see $P$ ).

ANG Angular distribution between or among particles in the final state. Includes also angular distribution involving decay products of not themselves explicitly listed. Includes angles used to study the decay of a system produced in the final state, even though the coordinate system axes may be defined with respect to the incident particles (e.g., Jackson angles, etc.) Also the equivalent, expressid as moments, etc.

MASS

Mass spectrum, $\mathrm{M}^{2}$ spectrum, or invariant cross section as a function of mass or mass squared.

PT Transverse momentum spectrum, $\mathrm{p}_{T}^{2}$ spectrum, or invariant cross section as a function of $\mathrm{p}_{\mathrm{T}}$. Does not include momentum transfer spectrum (see ANGP). Inciudes transverse mass $=\sqrt{p^{2} T+m^{2}}$, unless the particle mass $(m)$ is also variable. 
Appendix IV - Reaction Data Descriptors (cont'd)

Any function of outgoing momentum or energy not included in any of the above. Includes, $\mathrm{E}, \mathrm{y}$ (rapidity, also rapidity gaps), $\mathrm{x}\left(=\mathrm{p}_{\mathbb{H}} / \mathrm{p}_{\max }\right)$, $p_{1}$, or other momentum or energy variable.

(for proposals only) Experiment proposes to measure complete four-vectors, without specifying exactly what analysis of them will be

\section{AMPLITUDES}

Functions linear in the amplitudes (i.e., involving the phases).

Partial wave amplitudes. Includes formation partial waves and production partial waves. Any attempt to measure amplitudes of deflicte $J$ langula

Amplitude not decomposed into states of definite j. Re/Im ratio, helicity amplitude, etc.

\section{VARIABLES RELATED TO SEI}

Density matrix elements, including joint density matrix elements.

Final state spin-1/2 polarization measurement. Includes Wolfenstein spin rotation parameters. Includes measurement of asymmetry off a polarized target when it is equal to the finat state polarization.

Asymmetry in scattering off a polarized target and/or with a polarized beam (with exception of special case noted under POL).

\section{MULTIPLICITIES}

MULT Multiplicity distribution, its average, ratio, or moments. Generally used in association with final states of the form N(PRONG), N(HADRON), etc., so that the individual final states do not have to be listed.

\section{SYNOPSIS}

\begin{tabular}{|c|c|}
\hline & cross section. \\
\hline ANGP & $\mathrm{d} \sigma / \mathrm{dt}, \mathrm{d} \sigma / \mathrm{d} n, \mathrm{~d} \sigma / \mathrm{du}, \mathrm{dN} / \mathrm{dt}, \mathrm{etc}$ \\
\hline ANG & ang. dist. between particles in final state \\
\hline MASS & mass $_{3}$ mass $^{2}$ \\
\hline & $\mathrm{PT}_{\mathrm{T}}, \mathrm{PT}_{\mathrm{T}}^{2}, \mathrm{P}$ \\
\hline & $\begin{array}{l}x, y, P, E, \Delta y \\
\text { four-vectors (proposals only) }\end{array}$ \\
\hline PWA & production or formation partial wave analysis \\
\hline AMP & amplitudes not decomposed into states of definite $j$ \\
\hline & density matrix elements \\
\hline POL & final state spin $1 / 2$ polarization measurement \\
\hline ASYM & asymmetry in scaltering off polarized target \\
\hline MULT & multiplicity distribution, its average or other moments \\
\hline MASS*MASS & Daltiz plot, triangle plot \\
\hline ANGP*MASS & Chew-Low plot \\
\hline$P * P$ & longitudinal phase space plot, rapidity correlation contour plot \\
\hline PT*P & Peyrou plot \\
\hline ANCP*P & $\mathrm{d} \sigma / \mathrm{d} \Omega^{\mathrm{iab}}$ for several bins in $\mathrm{p}^{\text {lab }}$ \\
\hline
\end{tabular}

Appendix $V$ - Particle Properties Descriptors

Types of particle properties data are indicated by the following descriptors:

MASS Mass or mass difference

WTotal width, total rate, mean life. Also difference and ratios of these.

PW Partial widths, partial rate, as well as any ratio or product of these such as branching ratio or integrated cross section. Also upper limits on charge asymmetry of for $K_{L} \rightarrow \pi l u, \eta$ for $K_{L} \rightarrow \pi^{+} \pi^{-}, x+i y$ for $\left.K_{S} \rightarrow \pi^{+} \pi^{-} \pi^{0}\right)$.

MOM Electric moment, magnetic moment, charge radius, moment ratios.

DEC Weak or electromagnetic decay parameter as defined by Review of . Phs. $48, n 0.2$, pt.2, April 1976, Ch. VI: $\begin{array}{ll}\rho, \eta, \xi, \delta, h, \mid g_{A} / g_{V} l, \phi_{A V}, g_{S}, g_{T}, g_{P} & \text { for } \mu \text { decay } \\ \text { slopes } g \text { and slope difference } \sigma(C P \text { viol }) & \text { for } K \rightarrow 3 \pi\end{array}$ slopes $g$ and slope difference $\sigma$ (CP viol) for $K \rightarrow 3 \pi$ $\begin{array}{ll}\text { form factors } f_{+}, f_{-}, f_{0} ; \lambda_{+}, \lambda_{-}, \lambda_{0}, \xi, f_{S}, f_{T} & \text { for } K \rightarrow \pi l \nu \\ \text { CP violation parameter } x+i y & \text { for } K_{S} \rightarrow \pi^{+} \pi^{-} \pi^{0}\end{array}$ charge asymmetry $\delta \quad$ for $K_{\mathrm{L}} \rightarrow \pi \ell \nu$ CP viol. parameters $\eta_{+,-}, \eta_{00}, \phi_{+-}, \phi_{00}, \epsilon, \epsilon^{\prime}$ for $K_{L} \rightarrow \pi \pi$

$\Delta S \neq \Delta 0$ parameter $x \quad\left\{\right.$ for $K^{0} \rightarrow \pi^{+} l^{-} \nu$

charge asymmetry $\quad$ or $\bar{K}^{0} \rightarrow \pi^{-} l^{+} \nu$

$\lg _{A} / g_{V} \mid, \delta, \alpha, \beta, \gamma, \phi, \Delta \quad$ for baryon decay

ON Quantum numbers.

EX Existence (e.g., particle search or evidence for a new particle in a mass spectrum).

Appendix VI - Institution Abbreviations

AACH PHYS, INST, DER TECH. HOCHSCHULE

ACUS PHYSICAL INST, OF ACAD, OF SCIENCES

AECL ATOMIC ENERGY CENTER AT LAHORE

AERE ATOMIC ENERCY RES. ESTAB.

AICH AICHI EDUCATIONAL UNIV.

ALBA STATE UNIV. OF NEW YORK AT ALBANY

ALMA INSTITUTE FOR HIGH ENERGY PHYSICS

AMER AMERICAN UNIV HIC

ACADEMY OF MINING AND METALLURGY

ATDDLE EAST TECHNICAL UNIV.

ANL ARGONNE NAT. LAB.

ANUC AUSTRALIAN NATIONAL UNIV. AT CANBERRA

AOYA AOYAMA GAKNIN UNIV.

ATEN NUCLEAR RES. CENTRE DEMOKRITOS

ATEN NUCLEAR RES. CENT

BARI UNIY. DI BARI

BELG INST. INTERUNIV. DES SCI, NUC.

BERG FYSISK INSTITUTT

BERL INST. HOCHENERGIEPHYS. DAW

BERN UNIV. BERN

BENA UNIV. DI BOLOGNA

BIEL
STATE UNIV. OF NEW YORK AT BINGHAMTON
AACHEN, GERMANY

AARHUS, DENMARK

LAHORE, INDIA

HARWELL, BERKS., ENGLAND

BEDFORD, MASS., USA

TOYOTA, AICHI PREF., JAPAN

ALBANY, N. Y, USA

WASHINCTON DC USA

CRACOW, POLAND

AMSTERDAM NETHERLANDS

ANKARA, TURKEY

ARGONNE, ILL., USA

CANBERRA, AUSTRALIA

TOKYO, JAPAN

TUCSON, ARIZ, U USA

AUCKLAND, NEW ZEALAND

BARI, ITALY

BRUXELLES, BELGIUM

BERGEN, NORWAY

ZEUTHEN/BERLIN，DDR

BERN, SWITZERLAND

BOLOGNA, ITALY

BIELEFELD, GERMANY 
Appendix VI - Institution Abbreviations (cont'd)

\begin{tabular}{|c|c|}
\hline BI RM & RMI NGHAM UNIV. \\
\hline $\begin{array}{l}\text { BNL } \\
\text { BOHR }\end{array}$ & $\begin{array}{l}\text { BROOKHAVEN NATIONAL LAB. } \\
\text { NIELS BOHR INSTITUTE }\end{array}$ \\
\hline BONN & UNIV, BONN \\
\hline BOST & BOSTON UNIV. \\
\hline BRAN & BRANDEIS UNIV. \\
\hline $\begin{array}{l}\text { BRIS } \\
\text { BROW }\end{array}$ & $\begin{array}{l}\text { H. H. WILLS PHYS. LAE., U, OF BRISTOL } \\
\text { BROWN UNIV. }\end{array}$ \\
\hline BRUX & UNIY. LIBRE DE BRUXELLES \\
\hline $\mathrm{BUCH}$ & BUCHAREST STATE UNIV. \\
\hline BUDA & CENTRAL RESEARCH INSTITUTE OF PHYSICS \\
\hline BUFF & STATE UNIY, OF NEW YORK AT BUFFALO \\
\hline CAEN & LAB. DE PHYS. CORPUSCULA!RE \\
\hline CAMB & CAMBRI DCE UNIV. \\
\hline CAMP & UNIV. OF CAMPINAS \\
\hline ARL. & CARLTON UNIV. \\
\hline CARN & CARNEGIE-MELLON UNIV. \\
\hline CASE & CASE WESTERN RESERVE UNIV. \\
\hline CAVE & CAVENDISH LAB., CAMERIDGE UNIV. \\
\hline CDEF & COLLEGE DE FRANCE \\
\hline CEA & CAMBRI DGE ELECTRON ACCEL. \\
\hline CERN & EUROPEAN ORG. FOR NUC. RES. \\
\hline $\mathrm{CHIC}$ & UNIV. OF CHICAGO \\
\hline & CZECH. HI GHER TECH. INST. AT PRAGUE \\
\hline CINC & UNIV. OF CINCINNATI \\
\hline CIOW & CARNEGIE INST. OF WASHINGTON \\
\hline CIPP & CANADIAN INST. OF PART ICLE PHYS. \\
\hline CIT & CALIF. INSTITUTE OF TECHNOLOGY \\
\hline CLER & UNIV. DE CLERMONT-FERRAND \\
\hline & CANADI AN NAT IONAL RESEARCH $C$ \\
\hline COLO & UNIV. OF COLORADO \\
\hline COLU & COLUMBIA UNIV. \\
\hline CORN & CORNELL UNIV. \\
\hline $\cos U$ & COLORADO STATE UNIV. \\
\hline COUN & THE COOPER UNION \\
\hline CRAC & INST, FOR NUCLEAR RESEA \\
\hline & CHALK RIVER NUCLEAR LABORATORIES \\
\hline CUNY & CITY UNIV. OF NEW YORK \\
\hline CURI & LABORATOIRE JOLIOT -CURIE \\
\hline DARE & DARESBURY NUC. PHYS. LAB. \\
\hline DART & DARTMOUTH COLLEGE \\
\hline DELH & UNIV. OF DELHI \\
\hline DESY & DEUTSCHES ELEKTRONEN-SYNCH. \\
\hline DORT & UNIV. DORTMUND \\
\hline DSRL & DUPONT AND CO., SÁVANNAH RIVER LAB. \\
\hline DUKE & DUKE UNIV. \\
\hline DURH & UNIV. OF DURHAM \\
\hline DUUC & UNIVERSITY COLLEGE \\
\hline & UNIY. OF EDI NBURGH \\
\hline EFI & ENRICO FERMI INST. FOR NUCL. STUDIES \\
\hline EMMA & EMMANUEL COLLEGE \\
\hline EPOL & ECOLE POLYTECHNI QUE \\
\hline EREV & STATE ATOMIC ENERGY COMM. AT EREVAN \\
\hline ETHZ & SWISS FEDERAL I NST. OF TECHNOLOGY \\
\hline FIRZ & UNIV. DI FIRENZE \\
\hline FISK & FISK UNIV. \\
\hline FLOR & UNIV. OF FLORIDA \\
\hline FNAL & FERMI NATIONAL AC \\
\hline FON & \\
\hline FRAS & LA \\
\hline FREI & UNIV. FREI BURG \\
\hline FRIB & UNIY. DE FRI BOURG \\
\hline FSU & FLORIDA STATE UNIV. \\
\hline FUK & \\
\hline
\end{tabular}

BI RMI NGHAM, ENGLAND

UPTON, L.I. , N. Y. USA US

BONN, GERMANY

BOSTON, MASS., USA

BRISTOL, ENGLAND

PROVI DENCE, R. I . USA

BRUXELLES, BELGIUM

BUCHAREST, ROMANIA

BUFFALO $N$, Y USA

CAEN FRANCE , USA

CAMBRIDGE, ENGLAN

SAO PAULO, BRAZI

OTTAWA, CANADA

PITTSBURCH, PA., USA

CLEVELAND, OHIO, USA

CAMBRIDGE, ENGLAND

CAMBRIDCE, MASS, , USA

CHICAGO, SWITZERLAND

PRAGUE, CZECHOSLOVAKIA

CINCI NNATI, OHIO, USA

WASHI NGTON, D.C., USA

MONTREAL, CANADA

PASADENA, CALIF., USA

CLERMONT, CERRAND, , FANCE

TTAWA, CANADA

NEIY YORK, $N$, $Y$, USA

ITHACA, N. Y, USA

FORT COLLINS, COLO., USA

NEW YORK, N. Y., USA

CRACOW, POLAND

CHALK RIVER, CANADA

NEW YORK, N. Y., USA

PARIS, FRANCE

HANOVER, N. H., USA

DELHI, INDIA

HAMBURG, GERMANY

DORTMUND-HORNBRUCH, GERMANY

AIKEN, S.C. . USA

DURHAM, N. C., USA

DURHAM, ENGLAND

EDINBURGH, SCOTLAND

CHICAGO, SCO USA

BOSTON, MASS.: USA

PARIS, FRANCE

EREVAN, USSR

ZURICH, SWITZERLAN

FIRENZE, ITALY

GASHYILLE, TENN., USA BATAVIA, ILL., USA

UTRECHT, NETHERLANDS

FRASCATI, ITALY

FRIBOURG, SWITZERLAND

TALLAHASSEE, FLA., USA
Appendix VI - Institution Abbreviations (cont'd)

\begin{tabular}{|c|c|c|}
\hline GENO & UNIV. DI GENOVA & GENOVA, ITALYY \\
\hline $\begin{array}{l}\text { GESC } \\
\text { GEYA }\end{array}$ & GENERAL ELECTRIC R AND D CENTER & SCHENECTADY, N. Y, USA \\
\hline & UNIV. DE GENEVE & GENEVA，SWITZERLAND \\
\hline AS & UNIV. OF GLASGOW & CLASGOW, SCOTLAND \\
\hline RAZ & UNIV. GRAZ & GRAZ, AUSTRIA \\
\hline GREN & GRENOBLE UNIV. & GRENOBLE, FRANCE \\
\hline GRUM & GRUMMAN AEROSPACE CORP. & BETHPAGE, N. Y, USA \\
\hline GSCO & GEOLOGICAL SURVEY OF CANADA & OTTAFA, CANADA \\
\hline GUIL & UNIV. OF SURREY AT GUILFORD & GUI LFORD, SURREY, ENGLAND \\
\hline HAIF & TECHNION - ISRAEL INST. OF TECHNOLOGY & HAIFA, ISRAEL \\
\hline AMB & UNIV. HAMBURG & HAMBURG, GERMANY \\
\hline HANO & HANOI UNIV. & HANOI, VIETNAM \\
\hline HARV & HARVARD UNIV. & CAMBRIDGE, MASS., USA \\
\hline HAMA & UNIV. OF HATAII & HONOLULU, HAFAI I USA \\
\hline HEID & UNIV. HEIDELBERG & HEI DELBERG, GERMANY \\
\hline HELS & HELSINGIN YLIOPISTO & HELSI NKI, FINLAND \\
\hline HIRO & HI ROSHIMA UNIV. & HIROSHIMA, JAPAN \\
\hline Hous & UNIV. OF HOUSTON & HOUSTON, TEXAS, USA \\
\hline IASD & INST. FOR ADVANCED STUDIES AT DUBLIN & DUBLIN, I RELAND \\
\hline [BM & I NTERNATI ONAL BUSI NESS MACHI NES, I NC. & PALO ALTO, CALIF., USA \\
\hline ICUT & INTERNAT IONAL CHRISTIAN UNIV. AT TOKYO & TOKYO, JAPAN \\
\hline IFA & INST. FOR ATOMIC PHYSICS & BUCHAREST, ROMANIA \\
\hline I IT & ILLINOIS INST. OF TECH. & CHICAGO, ILL., USA \\
\hline ILL & UNIV. OF ILLINOIS & URBANA, ILL, USA \\
\hline ILLC & UNIV. OF ILLINOIS AT CHICAGO & CHICAGO ILL. USA \\
\hline IND & UNIV. OF INDIANA & BLOOMI NGTON, IND., USA \\
\hline I NNP & INST. FOR NUCL. PROBLEMS, ACAD. OF SCI. & MOSCOW, USSR \\
\hline INUS & INST. FOR NUCLEAR STUDY AT TOKYO UNIV. & TOKYO, JAPAN \\
\hline IOWA & UNIV. OF IOWA & IOWA CITY, IOWA, USA \\
\hline I PN & INST. DE PHYS, NUCLEAIRE & ORSAY, FRANCE \\
\hline I PNP & INSTITUT DE PHYSI QUE NUCLEAIRE & PARIS, FRANCE \\
\hline I RAD & INSTITUTE DU RADIUM & PARIS, FRA \\
\hline ISSP & SOLID STATE PHYS, INST., UNIY, OF TOKYO & TOKYO, JAPAN \\
\hline ISU & IOWA STATE UNIV. & AMES, IOWA, USA \\
\hline ITEP & INST. FOR TEOR. AND EXP. PHYS. & MOSCOW, USSR \\
\hline IUPU & INDIANA U. - PURDUE U. AT INDIANAPOLIS & INDI ANAPOL IS, IND., USA \\
\hline JAGL & JAGELLONIAN UNIY. & CRACOW, POLAND \\
\hline JAMU & JAMMU UNIV. & JAMMI-TAWI, I NDIA \\
\hline JHU & JOHNS HOPKINS UNIV. & BALT IMORE, MD. USA \\
\hline J [NR & JOINT INST. FOR NUCL. & DUBNA, USSR \\
\hline & UNIV. OF KANSAS & LAWRENCE, KANSAS, USA \\
\hline KARL & TECHNISCHE UNIV. KARLSRUHE & KARLSRUHE, GERMANY \\
\hline KEK & NAT. LAB FOR HIGH ENERGY PHYS, , JAPAN & TSUKUBA-GUN, JAPt \\
\hline KFAJ & KFA JULICH & BONN, GERMANY \\
\hline KHAR & PHYSICO-TECH. INST., ACAD. SCI , UKR.SSR & KHARKOY, USSR \\
\hline KI AE & KURCHATOV INST. OF ATOMIC ENERGY & MOSCOW, USSR \\
\hline KIEL & KIEL UNIV. & KIEL, GERMANY \\
\hline KII & KINKI UNIV. & OSAKA, JAPAN \\
\hline KNTY & UNIV. OF KENTUCKY & LEXINGTON, $K Y$, USA \\
\hline KOBE & KOBE UNIV. & KOBE, JAPAN \\
\hline KONA & V UNIV. & KOBE, JAPAN \\
\hline KWAN & KWANSAI GAKUIN UNIV & HYOGO-KEN, JAPAN \\
\hline & KYOTO UNIY. & KYOTO, JAPAN \\
\hline LALO & LINEAR ACCELERATOR LAB, ORSAY & ORSAY, FRANCE \\
\hline LANC & LANCASTER UNIV. & LANCASTER, ENGLAN \\
\hline LAPP & LAPP UNIV. & ANNECY, FRANCE \\
\hline LASL & ALAMOS SCIENTIFIC LAB. & LOS ALAMOS, N. M., USA \\
\hline LAUS & UNIV. OF LAUSANNE & LAUSANNE, SWITZERLAND \\
\hline $\begin{array}{l}\text { LBL } \\
\text { LEBD }\end{array}$ & U. C. LAWRENCE BERKELEY LAB. & BERKELEY, CALIF, , USA \\
\hline LEHI & LEBEDEV PHYSICS & MOScon \\
\hline $\begin{array}{l}\text { LEHI } \\
\text { LEID }\end{array}$ & $\begin{array}{l}\text { LEHIGH UNIV. } \\
\text { INST. LORENT }\end{array}$ & BETHLEHEM, PA., USA \\
\hline & INST. OF NUCL. PHYS & ETHERLANDS \\
\hline LINZ & LINZ INST ITUT FUR PHYSIK, KEPLER & LENI NGRAD, USS \\
\hline
\end{tabular}


Appendix VI - Institution Abbreviations (cont'd)

\begin{tabular}{|c|c|}
\hline IVP & LIVERPOOL UNIY. \\
\hline & LAWRENCE LIVERMORE LAB \\
\hline LOIC & I MPERIAL COL. OF SCI. AND TECH. \\
\hline LOKC & KING'S COLLEGE \\
\hline LOQM & QUEEN MARY COLLEGE \\
\hline LOUC & UNI VERSITY COLLEGE \\
\hline LOUG & LOUGHBOROUGH UNI $\mathrm{V}$. OF TECHNOLOGY \\
\hline LOWC & WESTFIELD COLLEGE \\
\hline LPGP & LAB, DE PHYS, GENERAL, UNIV. PARIS \\
\hline LPNP & LAB. DE PHYS. NUCL. ET HAUTES ENERGIES \\
\hline LRC & LEWIS RESEARCH CENTER, NASA \\
\hline LSU & LOUISIANA STATE UNIV. \\
\hline LUND & UNIY. I LUND \\
\hline LYON & INST. DE PHYS, NUCL., UNIY. DE LYON \\
\hline MADR & JUNTA DE ENERGIA NUCLEAR \\
\hline MANH & MANHATTAN COLLEGE \\
\hline MANI & UNIV. OF MANITOBA \\
\hline MANZ & UNIV. MAINZ \\
\hline MASA & UNIV. OF MASSACHUSETTS \\
\hline MASB & UNIY. OF MASSACHUSETTS \\
\hline MCGI & MCGILL UNIV. \\
\hline MCHS & UNIV. MANCHESTER \\
\hline MELB & UNIY. OF MELBOURNE \\
\hline MICH & UNIY, OF MICHIGAN \\
\hline MILA & UNIV. DI MILANO \\
\hline MINN & UNIV. OF MINNESOTA \\
\hline & MIAMI UNIV. \\
\hline MIRA & INTL. CENTER FOR THEO. PHYS., MIRAMARE \\
\hline MISU & MISSISSIPPI STATE UNIV, \\
\hline MIT & MASSACHUSETTS INST, OF TECHNOLOGY \\
\hline MODE & ISTITUTO DI FISICA DELLA UNIVERSITA \\
\hline MONS & UNIV. DE L'ETAT, MONS \\
\hline MOSU & MOSCOW UNIV. \\
\hline MPIH & MAX-PLANCK-INST. FUR PHYS,-ASTROPHYS. \\
\hline MPIM & MAX-PLANCK-INST. FUR PHYS.-ASTROPHYS \\
\hline & NASA MANNED SPACECRAFT CENTER \\
\hline MSNA & INS. DI FISICA DELL UNIV. \\
\hline MSU & MICHIGAN STATE UNIV. \\
\hline MTHO & MT . HOL YOKE COLLEGE \\
\hline MUDD & HARVEY MUDD COLLEGE \\
\hline MULH & CENTRE UNIV. DU HAUT-RHIN \\
\hline MUNI & NUNICH UNIV. \\
\hline NACO & NAGOYA UNIV. \\
\hline NANC & UNIY. DE NANCY \\
\hline NAPL & UNIV. DI NAPOLI \\
\hline NARA & NARA WOMEN'S UNIV \\
\hline NDAM & UNIV. OF NOTRE DAME \\
\hline NEAS & NORTHEASTERN UNIV. \\
\hline NEBR & UNIY. OF NEBRASKA \\
\hline NEUC & UNIV. OF NEUCHATEL \\
\hline NEVI & NEVIS LAB. \\
\hline NI JM & R. K. UNIV. NIJMECEN \\
\hline NILU & NORTHERN ILLINOIS UNIY \\
\hline NORD & NORDISK INS. FOR TEOR. \\
\hline Novo & INST, OF NUCL. PHYS. \\
\hline NRL & NAVAL RESEARCH LABORATORY \\
\hline NSF & NATIONAL SCIENCE FOUNDATION \\
\hline NWES & NORTHWESTERN UNIY. \\
\hline NTU & \\
\hline OBER & OLLEGE \\
\hline $\mathrm{OHIO}$ & OHIO UNIV. \\
\hline OREG & UNIV. OF OREGON \\
\hline ORNL & OAK RIDGE NATIONAL LAB. \\
\hline
\end{tabular}

LIVERPOOL, ENGLAND

IORMORE, CALIF.., USA

LIVERY, ENGLAND

ONDON, ENGLAND

LONDON, ENGLAND

LUGHBOROUGH, ENGLAND

LONDON, ENGLAND

PARIS, FRANCE

PARIS, FRANCE

BATON ROUGE, LA., USA

ILLEURBANNE, FRANCE

MADRID, SPAIN

EEW YORK, N. Y., USA

WINNI PEC, CANADA

MAINZ, GERMANY

AMHERST, MASS., USA

MSTON, MASS. . USA

MANCHESTER, ENGLAND

AARKVLLE, AUSTRALIA

ANN ARBOR, MICH., USA

MI LANO, ITALY

MNNEAPOLIS, MINN., USA

OXFORD, OHIO, USA

STATE COLLEGE, MISS, USA

CAMBRIDGE MASS MS

MONS, BELGIUM

MOSCOW, USSR

MEIDELBERG, GERMANY

MUNICH, GERMANY

MESSIN, TEXAS, USA

SOST LANSINC,

SOUTH HADLEY, MICH., USA

CLAREMONT, CALIF. USA

MULHOUSE, FRANCE

MUNICH, GERMANY

NAGOYA, JAPAN

NANCY, FRANCE

NAPOL, ITALY

NOTRE DAME, IND., USA

BOSTON, MASS.., USA

LINCOLN, NEBR, USA USA

RVI NGTON-ON-HUDSON, Y.Y, , USA

NI JMEGEN, NETHERLANDS

DEKALB, ILL. USA

NOVOSI BI RSK, USSR

ASHI NGTON, D.C. . USA

MASHI NGTON, D.C. USA USA

EAANSTON, ILL.. USA

NEW YORK, N. Y., USA

OBERLIN, OHIO, USA

ATHENS, OHIO, USA

OAK RIDGE, TENN., USA
Appendix VI - Institution Abbreviations (cont'd)

ORSA UNIV, DE PARIS, FAC. DES SCI.

OSAKA UNIY.

OSMANIA UNIV.

OSMA OSMANA UN

OSSE SCI. EDUC. INST.

OTTA UNIV. OF OTTAWA

OXF OXFORD UNIV.

PADO UNIV. DI PADOVA

PARM UNIV. OF PARMA

PATR UNIV. OF PATRAS

PENN UNIV. OF PENNSYLVANIA

PISA UNIV DI PISA

PITT UNIV. OF PITTSBURGH

PPA PRINCETON-PENN, PROTON ACCEL

PRAG INSTITUTE OF PHYSICS, CSAY

PRIN PRINCETON UNIV

PSU PENNSYLVANIA STATE UNIV.

PTIU PHYSICAL TECH. INST. OF ACAD. OF SCI

PURD PURDUE UNIV.

QUB QUEENS UNIV. AT BELFAST

QUNS QUEENS COLLEGE OF CUNY

RAMA TEL-AVIV UNIV. AT RAMAT AVIV

REED REED COLLEGE

REHO HEIZMANN INST. OF SCI.

RHEL RUTHERFORD HICH ENERGY LAB

RICE WILLIAM MARSH RICE UNI

RMCS ROYAL MILITARY COLLEGE OF SCIENCE

ROCH UNIV. OF ROCHESTER

ROCK ROCKEFELLER UNIV

ROMA UNIV. DI ROMA
RUTG RUTCERS UNIV.

RUTG RUTGERS UNIV.

SAGA SAGA UNIV.

SAIT SAITAMA UNIY

SANT UNIV. DE SANTANDER

SCST STATE COMM. ON SCIENCE AND TECH.

SEATTLE PACIFIC COLLEGE

INST. OF HICH EN. PHYS.

SETON HALL UNIV.

UNIV OF SOEFTELD

SIEGEN UNIV.

STANFORD LINEAR ACCEL. CENTER

SOUTHEASTERN MASSACHUSETTS UNIV.

SOUTHERN METHODIST UNIY.

ISRAEL AEC SOTEG. NUCL. RESEARCH CENTER SCUOLA NORMALE SUPERI ORE HICH INST. OF CHEM. TECH.

BULGARIAN ACAD. OF SCI.
STANFORD RESEARCH INST.

STANFORD RESEA

UNIV OF STELLENBOSCH

STEVENS INST. OF TECH.

ST. LOUIS UNIV.

STATE UNIY. OF NEW YORK AT STONYBROOK

CENTRE DES RES. NUCLEAIRES
ORSAY, FRANCE

OSAKA, JAPAN

HYDERABAD, I NDIA

COLLAMBUU, JHAPA OHIO, USA

OTTAWA, CANADA,

OXFORD, ENGLAND

PADOVA, ITALY

PARMA, ITALY

PATRAS, GREECE

PAVIA, ITALY

PITTSBURCH,

PRINCETON, PA., USA

PRAGUE, CZECHOSLOVAKIA

PRINCETON, N. J., USA

UNIVERSITY PARK, PA., USA

TASHKENT, USSR

LAFAYETTE, IND., USA
BELFAST, NORTHERN IRELAND

MONTREAL. CANADA

FLUSHING, N. Y., USA

PORTLAND, OREGON, USA

REHOVOTH, ISRAEL

CHILTON, DID., OXON., ENGLAND

HOUSTON, TEXAS, USA

ROSKILDE, DENMARK

ROCHESTER, $N$ Y Y USA

NEW YORK, $N, Y$, USA

NEW BRUNSWICK, N. J., USA

GIF-SUR-YVETTE, FRANCE

SAGA, JAPAN

SAITAMA, JAPAN

SANTANDER, SPAIN

HANOI, NORTH VIETNA

SEATTLE, WASH. , USA

SERPUKOV, USSR

SOUTH ORANGE, N. J., USA

SHEFFI ELD, YORKS. , ENGLAND

SOUTAAMPTON, ENGLAND

STANFORD, CELIF. USA

NORTH DARTMOUTH, MASS, USA

DALLAS, TEXAS, USA

YAVNEH, ISRAEL

PISA, ITALY

SOFIA, BULGARIA

SOFLA, BULGaRIa

MENLO PARK, CALIF., USA

CAPE PROVINCE, SOUTH AFRICA

HOBOKEN, N. J.., USA

STOCKHOLM, SWEDEN

STONYBROOK, L.I., N. Y., USA

STRASBOURG, FRANCE
SUFFOLK, ENGLAND 
Appendlx $\mathrm{YI}$ - Institution Abbreviations (cont'd)

SUSS SUSSEX UNIV.

UNIV OF SYDNEY

SYRA SYRACUSE UNIY.

TASMANIA AND M UNIV.

TATA TATA INST. OF FUNDAMENTAL RESEARCH

TBIL

TELA

TEMP

TENN

TIV. OF TENNESSEE

TOKYO METROPOLITAN UNI

TOGA TOHOKU-GAKUIN UNIV

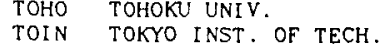

TOIN TOKYO INST. OF
TOKY UNIV. OF TOKYO

TRIUMF, UNIV. OF BRITISH COLLMBIA

TSOP SOPHIA UNIV.

TUAT TOKYO UNIV, OF AGRICULTURE AND TECH.

TUED TOKYO UNIV. OF EDUCATION

TUFT TUFTS UNIV.

UATH UNIV. OF ATHENS

UBEL UNIY OF AELCRADE

UCB UNIV. OF CALIF. AT BERKELEY

UCD UNIV. OF CALIF. AT DAVIS

UCLA UNIV. OF CALIF. AT LOS ANGELES
SUSSEX, ENGLAND SYRAY'A AUST Y USA COLLEGE STATION, TEXAS, USA HOBART TASMANIA, AUSTRALIA BOMBAY, INDIA TBILSI, USSR TEL-AVIV, ISRAEL PHILADELPMIA, PA., USA RNOXVILLE, TENN., USA AUSTIN, TX, USA TOKYO, JAPAN TORONTO, CANADA MIYAGI, JAPAN SENDAI, JAPAN TOKYO, JAPAN TOKYO, JAPAN VANCOUVER CANADA TRIESTE, ITALY TRIESTE, ITALY TOKYO, JAPAN TOKYO, JAPAN MEDFORD, MASS., USA TOKYO, JAPAN

ATELNS, GREECE BERKELEY, CALIF., USA DAVIS, CALIF., USA IRVINE, CALIF, USA LOS ANGELES, CALIF., USA OAK RIDGE, TENN., USA RIVERSIDE, CALIF., USA SANTA BARBARA, CALIF U, USA SANTA CRUZ, CALIF., USA ULAN-BATOR MOL, USA MADRID, SPAIN COLLECE PARK, MD. . USA SCHENECTADY, $N$. Y., USA ALBUOUEROUE, NEW MEX., USA EAST ORANCE, N. J., USA LOS ANGELES, CALIF., USA SALT LAKE CITY, UTAH, USA UTRECHT, NETHERLANDS UTSUNOMIYA, JAPAN UPPSALA, SWEDEN VALENC:TA, SPAIN NASHVI LLE, TENN., USA VARANASI, INDIA POUCHKEEPSIE, N. Y., USA CHANA, AUSTRIA VIEVMA, AUSTRIA, VA., USA WAKAYAMA-SH VA, USA WARSAW, POLAND SEATTLE, WASH., USA
DETROIT, MICH., USA LONDON. CANADA

Appendix VI - Institution Abbreviatlons (cont'd)

WILL COLLEGE OF WILLIAM AND MARY

WISC UNIV. OF WISCONSIN

WOOD UNIV. OF THE WITWATERSRAND

WOPI WORCESTER POLYTECHNIC INST.

WUPP UNIV. WUPPERTAL

WUSL WASHINGTON UNIV.
WYOM UNIV. OF WYOMING

YALE YALE UNIY.

YERE YEREVAN PHYSICS INST

ZACR INSTITUTE RUDER BOSKOVIC, ZAGREB

ZEEM ZEEMAN LAB. UNIV. OF AMSTERDAM

UNKNOWN INST UTUTION
WILLIAMSBURG, VA., USA MADISON, WISC, USA
JOHANNESBURG, SOUTH AFRICA JOHANNESBURG, SOUTH AFRIC
WOODSTOCK, MD. USA WORCESTER, MASS., USA WUPPERTAL, GERMANY ST LOUIS, MO USA LARAMIE, WYOMING, USA NEW HAVEN, CONN. USA YEREVAN, ARMENIA, USSR YOKOHAMA, JAPAN ZAGRE, YUGOSLAVIA

\section{Appendix VII - Particle Abbrevlations \\ $A D$ \\ ADEL (1232P33)0 ADEL $(1550531) 0$ ADELL (1570D33)0 ADEL (1690P33)0 ADEL $(1890 \% 35) 0$ ADEL $(1900531) 0$ $\operatorname{ADEL}(1950 \mathrm{~B}) 0$ ADEL (1950F37)0 ADEL (1960D35) ADEL (2420B) 0 ADEL $(2420 \mathrm{H} 311) 0$ $\operatorname{ADEL}(2850 B) 0$ ADEL ( $3230 B) 0$ $A D * 0$ \\ $A G$ \\ AHVY-LEPTON AHYY-LEPTON AKAPPAO
AKN $(I=0)$ \\ $\operatorname{AKN}(I=1)$ \\ AKN $(1660) 0$ \\ ADEL (1232P33)+ ADEL(1232P33)- ADEL(1232P33)-- ADEL(1670D33)+ $\quad$ ADEL(1650S31)- $\quad$ ADEL(1650531)-- ADEL (1690P33)+ ADEL(1690P33)- ADEL (1690P33)-- ADEL(1890F35)+ ADEL(1890F35)- ADEL(1890F35) -- ADEL(1900S31)+ ADEL(1900531)- ADEL(1900531)-- ADEL (1910P31)+ ADEL(1910P31)- ADEL(1910P31)-- ADEL (1950B)+ + ADEL(1950B)- ADEL(1950B)-- ADEL $(2150)+$ ADEL $(1960)-$ ADEL $(1960035)$ $A D E L(2420 B)+\quad A D E(2160)-1203)-\quad A D E L(2160)-\infty$ $A D E L(2420 \mathrm{H} 311)+$ ADEL $(2420 \mathrm{H} 311)-\mathrm{ADEL}(2420 \mathrm{H} 311)$ $\operatorname{ADEL}(2850 \mathrm{~B})+\quad \mathrm{ADEL}(2850 \mathrm{~B})-\quad$ ADEL $(2850 \mathrm{~B})-$ ADEL (3230B)+ ADEL(3230B)- ADEL(3230B) -- \\ $C=$ SILVER NUCLEUS \\ $C=$ ANT I-HELI IMS-4 NUCLEUS \\ C= GENERAL ANTI HEAVY-LEPTON \\ $C=$ GENERAL ANT \\ $C=$ ANTI-K NUCLEON I=O INITIAL STATE (AND ELASTIC FINAL STATE) \\ C= ANTI-K NUCLEON I 1 INITIAL STATE (AND ELASTIC FINAL STATE). DO NOT USE FOR THE SINGLE
PHYSICAL STATE (K- N) \\ $C=$ POSSIBLE K- OMECA STATE} $\operatorname{AKN}(1700) 0$ AKPRIME (1400)0

AKPRIME (1400)AKO

AK* (UNSPEC) AK* (UNSPEC) $A K^{*}(1420) 0$ $A K^{*}(1660) 0$ $A K *(2200) 0$ $A K^{*}(2800) 0$ $A K^{*}(892) 0$
$C=J P=0-$ STATE SEEN IN PWA BY BRANDENBURG 76 ,

$C=J P=0-S T A T E D O M I N A T L Y$ INTO EPSILON KO DECAYING PREDOMINATLY INTO EPSILON KO 
ALAMBDA

ALAM( $1330 B)$
ALAM( 1405501$)$

ALAM(1520D03)

ALAM( 1600 PO1)

ALAM(1670S01)

ALAM (1690003)

$\operatorname{ALAM}(1800609)$

ALAM (1800PO)

ALAM( $(800501)$

ALAM( $(1860003)$

ALAM(1870SO1)

$\operatorname{ALAM}(2010)$

$\operatorname{ALAM}(2020507)$

ALAM (2100B)

ALAM( 2100G07)

ALAM(2110F05)

ALAM (2350B)

ALAM $(2585 B)$

AN

$\operatorname{ANN}(I=1)$

ANU

ANUCLEON

ANYTHI NG

AN (1470B) 0 AN (1470P11)0 AN (1520B) 0

AN(1535S11)0

AN $(1670015) 0$

AN (1688F15)0

AN $(1700 B) 0$

AN (1700D 13)0

AN $(1700511) 0$

AN (1780P11)

AN (1810P13)0

AN (2000F 15$) 0$

N $(2040 D 13) 0$

AN $(2100015) 0$

$\operatorname{AN}(2100511) 0$

AN (2190B)O

AN (2 190G17)0

AN $(2220 \mathrm{H} 19) 0$

AN (3030B)O

AN $(3245 B) 0$

AN (3690B)O

$\operatorname{AN}(3755 B) 0$

AOMEGA

AP

AOLOW (1240)0
$C=A N T I-L(1770) 0$

$C=$ ANTI-NUCLEON NUCLEON I $=0$ INITIAL STATE (AND

ELASTIC FINAL STATE LLAST IC FINAL STATE), DO NOT USE FOR THE SINGLE PHYSICAL STATE (AP N)

$C=$ ANTI-NUCLEON

I-NUCLEUS

ANUMU

FOR USE IN INCLUSIVE REACTIONS, ALSO FOR $A N(1470 B)-$

AN (1470P11)

AN(1520B)-

AN(153511)-

AN(150015)-

AN $(1688 \mathrm{~F} 15)-$

AN (1700B)-

AN(1700D13)-

AN(1700511)-

AN (1780P11)-

AN $(1810 \mathrm{P} 13)-$

AN(2000F15)-

AN (2040013)-

AN(2100D15)-

AN(2100S11)-

AN (2190B)-

$A N(2190 \mathrm{G} 17)-$

AN (2550B)-

AN (2650B)-

AN (3245B)

AN (3690B)

AN(3755B)-

$C=$ ANTI-QUARK
AO $(1240-1400) 0$

ASI CMAO

ASI G (1385P13)0

ASIG $(1440 \mathrm{~B}) 0$

ASIG (1580D 13)0

ASIC (1520B)0

ASIG $(1620811)$

ASIG (1620S11)0

ASIG (1670B) O

ASIG(1670D13)O

ASIG (1690B) 0

ASIG $(1750 S 11) 0$

ASIG (1765D 15)0

ASIC(1800P11)

ASIG (1915B)0

ASIG (1915F 15)0

ASIG(1940D13)0

ASIC (2000S11)0

ASIG (2030B) 0

ASIG (2030F 17$) 0$

ASIG(2080 P13)

ASIG(2100 17$) 0$

ASIG (2210H111)0

ASIG(2215G19)0

ASIG (2250B) 0

ASIG(2260D15)0

ASIG (2455B)0

ASIG $(3000 B) 0$

AT

$\operatorname{AXI}(1530 P 13) 0$

$\operatorname{AXI}(1530) 0$

AXI $(1820) 0$

AXI ( 1940$) 0$

AXI $(2030) 0$
AXI $(2250) 0$

AXI (2250)0
$\operatorname{AXI}(2500) 0$

AX1 ( 1100$) 0$

A1. $5(1170) 0$

A2 $(1310) 0$

$\mathrm{A} 3(1640) 0$

A4 (1900)0

${ }_{B 1}^{B R}(1040) 0$

$B(1235) 0$

$\mathrm{C}$ CC

CD

CHARGED+

CHARM

CHGD-HADRO

CHI $(3410) 0$
CHI $(3530) 0$
ASI GMA +

ASI GMA-

ASIC(1385P13)+ ASIG(1385P13)-

ASIG $(1440 B)+$

ASIG $(1480 B)+\quad$ ASIG $(1480 B)-$

ASIG (1580B + ASIG(1580D13)-

ASIG(1620P11)+ ASIG(1620P11)

ASIC(1520S11)-

ASIC $(1670 \mathrm{~B})+$

ASIG(1670D13)+ ASIG(1670D13)-

ASIG(1690B)+ ASIG(1690B)-

ASIC(1750S11)+ ASIG(1750511)-

ASIC $(1840 \mathrm{P} 13)+$ ASIG(1765D15)-

ASIC $\left(1880 P_{11}\right)+$ ASIG(1840P13)-

ASIC (1915B)+ ASIG $(1915 B)-$

SIC $\left(1915 F_{15}\right)+$ ASIG $\left(1915 F_{15}\right)$

ASIG (1940D13)-

ASIG(2000S11)+ ASIG(2000S11)-

ASIG (2030B $)+$ ASIG(2030B)-

ASIC $2030 \mathrm{~F} 17)+$ ASIG(2030F17)-

ASIG(2080P13)+ ASIG(2070F15)-

ASIG $(2100 \mathrm{C} 17)+$ ASIG(200P13)-

ASIG(2210H111)+ ASIG(2310H111)-

ASIG $(2215 \mathrm{C19})+$ ASIC $(2215 \mathrm{C19})-$

ASIG(2250B)+ ASIG(2250B)-

ASIG(2260D15)-

ASIG (2455B)+ ASIG(2455B)-

ASIG(3000B)+ ASIG(2620B)-

$\mathrm{C}=$ ANTI-TRITIUM NUCLFUS

$\operatorname{AXI}(1530 P 13)+$

AXI $(1630)+$

$\operatorname{AXI}(1940)+$

$\operatorname{AXI}(2030)+$

$\operatorname{AXI}(2500)+$

A1. $5(1170)+\quad$ A1 $(1100)-$

AZ $(1310)+$ A2(1310)-

$\begin{array}{ll}\mathrm{A} 3(1640)+ & \mathrm{A} 3(1640)- \\ \mathrm{A} 4(1900)+ & \mathrm{A} 4(1900)-\end{array}$

CHEMICAL SYMBOL

$B 1(1040)+$

B1 (1040)-

C= CARBON NUCLEUS

$C=$ CHARGE CONJUGATE REACTION

$C=$ CADMIUM NUCLEUS

CHARGED PARTICLE. UNLIKE PRONG, THIS DOES NOT

C POSLCE POSSIBLE NEUTRALS

C= NOSTIVE CLARGED PARTICLE

$C=$ CHARMED PARTICLE

$\mathrm{C}=$ CHARGED HADRON 
$\cos M I C$
$\mathrm{CU}$

C*(4.44)

DD

DELTA 990$) 0$ DEL (UNSPEC)O

DEL (UNSPEC) +

DEL (UNSPEC)++

DEL (UNSPEC)-

$\operatorname{DEL}(1232 P 33) 0$ DEL (1650S31)0

DEL (1690P33)0

DEL (1890F 35$) 0$

DEL (1900S31)O

DEL (1950B)O

DEL(1950F37)O

DEL ( 1960D35)0

DEL (2160)0

DEL $(2420 H 311) 0$

$\operatorname{DEL}(2850 \mathrm{~B}) 0$

DEL (3230B)

D*0

D(1285)

EPSILON $(1200)$

EPSILON(700)

ETA

ETAPRIME

XOTI C-HYPERON

EXOTIC-MESON

EXOTIC-NUCLEON

$\operatorname{EX}(1640) 0$

$\operatorname{Ex}(1640)++$

Ex(1640)+++

EX (1640)

E+

$E(1420)$

F

FE

FPRIME

FRAG

FRAGB

F1 (1540)0

GAMMA
Appendix VII - Particle Abbrevlations (cont'd)

$C=$ COSMIC-RAY PARTICLE OF UNDETERMINED NATURE $C=$ COPPER NUCLEUS

$C=$ DIFFRACTION DISSOCLATION. TO BE FOLLOWED BY NAMES OF PARTICLES WHICH WERE PRODUCED VIA DELTA $(970)+\quad$ DELTA $(970)-$

$C=[=3 / 2$ BARYON OF UNSPECIFIED MASS, USE PRIMARILY

$C=I=3 / 2$ BARYON OF UNSPECIFIED MASS, USE PRIMARILY

IN PROP, RVUE, COMP

PSECIFIED MASS, USE PRIMARILY

I =3/2 BARYON OF UNSPECIFIED MASS, USE PRIMARILY

IN PROP, RVUE, COMP

DEL (1232P33)+ DEL(1232P33)++ DEL(1232P33)-

DEL $(1550531)+$ DEL $(1650531)++$ DEL $(1650531)-$

DEL $(1570033)+$ DEL (1670D33)++ DEL $(1670033)-$

DEL $(1690 \mathrm{P} 33)+$ DEL (1690P33)++ DEL(1690P33)-

DEL $(1900531)+$ DEL $(1900531)++$ DEL $(1900531)-$

$\mathrm{DEL}(1910 \mathrm{P} 31)++$ DEL $(1910 \mathrm{P} 31)++$ DEL $(1910 \mathrm{P} 31)-$

DEL $(1950 \mathrm{~B})+\quad$ DEL $(1950 \mathrm{~B})++$ DEL $(1950 \mathrm{~B})-$

DEL (1950F37)+ DEL(1950F37)++ DEL(1950F37)-

DEL(1960D35)+ DEL(1960D35)++ DEL(1960D35) -

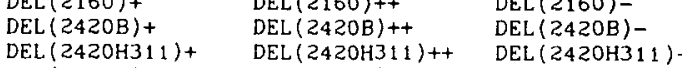

DEL $(2420 \mathrm{H} 311)+$ DEL $(242 \mathrm{OH} 311)++$ DEL $(242 \mathrm{OH} 311)$

DEL (3230B $++\quad$ DEL (3230B $)++\quad$ DEL (2850B) -

${ }^{\text {C }}=$ LOW MASS D PI PI BUMP

$C=$ PIPI S-WAVE (NEAR 1200 MEV)
$C=$ PIPI S-WAVE (NEAR 700 MEV)

= CANNOT BE FORMED OF QUARK-ANT I-QUARK OR DQO

$C=$ CANNOT BE FORMED OF QUARK-ANT I-OUARK

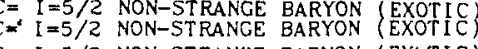

$C=I=5 / 2$ NON-STRANGE BARYON (EXUTIC)

C= $=5 / 2$ NON-STRANCE BARYON (EXOTIC)

$C=I=5 / 2$ NON-STRANGE BARYON (EXOTIC)

$C=$ POSITRON

$=$ ELECTRON

$C=F(1270)$ MESON RESONANCE

$C=$ I RON NUCLEUS $C=$ FLUORINE NUCLEUS - - NOTE NAME IS NOT SAME AS

C= FRAGMENT FROM NUCLEUS, USED FOR DESCRIPTION OF

HEAVY TON EXPERIMEMT

$C=$ FRAGMENT OF TARGET

$\mathrm{F} 1(1540)+$ F1(1540)-

$\mathrm{C}=$ TWO OR MORE GAMMAS
CAMMA (S)

$\mathrm{C}(1680) 0$

HADRON

HADRONO

HADRON+

HADRON-

HADRON(S)

$\mathrm{HE}$

HNUCLEUS

HVY-LEPTON

HYY-LEPTON+

HVY-LEPTON-

HYPERON

$\mathrm{H}(2040)$

H(990)
INELASTIC

J/PSI $(3100) 0$

KAPPA $(1250)+$

KAPPA (1250)-

$\mathrm{KN}(I=0)$

$\mathrm{KN}(I=1)$

$\operatorname{KN}(1660) 0$

KN(1700)0

$\mathrm{KN}(1700)$

$\operatorname{KPI}(530)++$

KPRIME (1400)0

KPRIME $(1400)+$

KS

K* (UNSPEC)

K* (UNSPEC) 0

$\mathrm{K}^{*}(\mathrm{UNSPEC})+$

$\mathrm{K}^{*}(\mathrm{UNSPEC})$
$\mathrm{K}^{*}(1225) 0$

$\mathrm{K}^{*}(1420) 0$

$\mathrm{K}^{*}(1660) 0$

$K *(2800) 0$

$\mathrm{K} *(892) 0$

LAMBDA

LAM(UNSPEC)

LAM(1330B)

$\operatorname{LAM}(1405501)$

LAM(1520D03)

LAM(1600P01)

LAM(1670501)

LAM (1800G09)

AM (1800P0 1$)$

LAM(1815F05)
Appendix VII - Particle Abbreviatlons (con('d)

$C=$ ONE OR MORE GAMMAS
G(1680)

C(1680)+ $\mathrm{C}(1680)$ -

= SINCLE HADRON, ANY CHARGE OR MASS

$C=$ TWO OR MORE HADRON

$C=$ CHARGE + HADRON

$\mathrm{C}=$ CHARGE - HADRON

$C=$ ONE OR MORE HADRONS

$C=$ HELIUM-4 NUCLEUS

$C=$ HELIUM 3

$C=$ HYPER-NUCLEUS

$C=$ GENERAL HEAVY LEPTON

$C=$ HEAVY LEPTON

$C=$ HEAVY LEPTON

$\mathrm{C}=$ GENERAL HYPERON

$C=[=0, J P=4+$ MESON RESONANCE

$C=$ SAME AS ANYTHING, EXCEPT ELASTIC EXCLUDED

$C=$ IRIDIUM NUCLEUS

$C=K P I$ S-WAVE

$C=K P I$ S-WAVE

$C=K$ LONG

$C=K$ NUCLEON I $=0$ INITIAL STATE (AND ELASTIC FINAL

STATE)

$C=K$ NUCLEON I=1 INITIAL STATE (AND ELASTIC FINAL STATE) DO NOT USE FOR THE SINCLE PHYSICAL

$\operatorname{KN}(1560)+$ KN(1660)-

$C=$ POSSIBLE K- OMEGA STATE

$C=$ POSSIBLE K- OMEGA STATE

$C=K$ PI S-WAYE STATE (EXOTIC)

$C=J P=0-$ STATE SEEN IN PWA BY BRANDENBURG 76 ,

DECAYING PREDOMINATLY TO EPSILON KO

$C=J P=0$ - STATE SEEN IN PWA BY BRANDENBURG 76

= $K$ SHORT PREDOMINATLY INTO EPSILON KO

$\begin{array}{ll}K^{*}(1225)+ & K^{*}(1225)- \\ K^{*}(1420)+ & K^{*}(1420)- \\ K^{*}(1660)+ & K^{*}(1660)- \\ K^{*}(2200)+ & K^{*}(2200)- \\ K^{*}(2800)+ & K^{*}(2800)- \\ K^{*}(892)+ & K^{*}(892)-\end{array}$

$C=I=0, S=-1$ BARYON RESONANCE, FOR USE PRIMARILY IN $C=$ BUMP, RVUE, COMP 
LAM(1830D05) LAM $(1860 P 03)$ $\operatorname{LAM}(2010)$ $(20)$
$(200 B)$ LAM (2100607) LAM(2110F05) LAM(2350B) $\operatorname{LAM}(2585 \mathrm{~B})$ LONGLI VED

$L(1770) 0$

MESON (1970)0

MESON $(1970)+$

MESON(2190)0

MESON $(2190)+$

$\operatorname{MESON}(2190)-$

$\operatorname{MESON}(3270) 0$

$\operatorname{MESON}(3500) 0$

$\operatorname{MESON}(3520) 0$

MM. GE. 0

MM. GE.

MONOPOLE

$\mathrm{MU}+$

$M(1033)$

$M(940)$

$M(953)$

NANO (2375)

NE NEUTRAL

NEUTRAL(S)

NIT

$\mathrm{NN}(\mathrm{I}=0$
$C=$ BUMP AT $2100 \mathrm{MEV}$

$C=[=0, \quad Y=0 \quad$ BUMPS

$C=$ LITHIUM NUCLEUS

STABLE UNDER STRONG OR E- DECAY; MASS AND OTHER $\mathrm{L}(1770)+$ NUMBERS NOT WELL-DETERMINED

$C=$ TWO OR MORE $L(1770)$

$C=1970$ MEV $I=1$ MESON DECAYING INTO $K^{*}(1420)$ AK

$C=1970$ MEV $I=1$ MESON DECAYING INTO $K *(1420)$ AK

$C=2190 \mathrm{MEV} I=1$ MESON OBSERVED IN AP P AND AP D

TOTAL CS

$C=2190 \mathrm{MEV} I=1$ MESON OBSERVED IN AP $P$ AND AP D

$C=2190$ MEV $I=1$ MESON OBSERVED IN AP P AND AP D

$C=$ NEW POSSIBLE PARTICLE OBSERVED IN E+ E- $\rightarrow$ MU MU- 2GAMMA AS A BUMP IN ZGAMOA MASS REPORTED IN SLAC-PUB-1644

$C=$ NEW POSSIBLE PARTICLE OBSERVED IN E+ E- $->$ MUt MU- ZGAMMA AS A BUMP IN ZGAMMA MASS REFORTED IN SLAC-PUB- 1644

$C=$ OBSERVED IN E+ E- 2GAMMA FINAL STATE IN WHICH FOR DETAIL A DUMP AT ISO MEV, SLE R-DESY-75-20

$C=$ TWO OR MORE UNDETECTED NEUTRAL PARTICLES (THIS IS THE STANDARD MEANING OF MISSING MASS IN BUBBLE CHAMBER EXPERIMENTS) ** SEE MM. GE.O AND

MM.GE. 1 FOR OTHER MISSING MASS STATES

$C=$ ZERO, ONE OR MORE UNDETECTED NEUTRAL PARTICLES SEE MM AND OR L FOR OTHER MISSING MASS

$C=$ ONE OR MORE UNDETECTED NEUTRAL PARTICLES ** SEE $C=$ MAGNET IC MONOPOLE

MU-

$C=$ NON-STRANGE, I=0 MESON RESONANCE

$C=$ NON-STRANGE, $I=0$ MESON RESONACE

C= NON-STRANGE, I=O MESON RESONNCE

$C=$ NEUTRON

$C=$ NUCLEON-ANT I NUCLEON I $=0$ BLMP

$\mathrm{C}=$ NEON NUCLEUS

$C=$ NUCLEON EPSILON $I=1 / 2$ FINAL STATE

$C=$ TWO OR MORE NEUTRAL PARTICLES -- DO NOT USE FOR

$C=$ ONE OR MORE NEUTRAL PARTICLES -- DO NOT USE FOR $C=$ NITROGEN

NUCLEUS -- NOTE NAME IS NOT SAME AS CHEMICAL SYMBOL

$=$ NUCLEON NUCLEON I=O INITIAL STATE (AND ELASTIC
FINAL STATE)
$\mathrm{NN}(I=1)$

NONSTRC-PRONC

NON-RES

$\mathrm{NRHO}(I=1 / 2)$

$\mathrm{RHO}(I=3 / 2)$

NUCLEUS

NUE

$N * 5 / 2$ (UNSPEC)

$N^{*}$ (UNSPEC)

$\mathrm{N}^{*}$ (UNSPEC) 0

$N^{*}($ UNSPEC $)+$

N* (UNSPEC)

N(CHARGED)

$N($ CHGD-HADRON )

N(FRAG)

N(GAMMA)

$N(H E)$

$\mathrm{N}(\mathrm{KO})$

$\mathrm{N}(\mathrm{K}-)$

N(LAMBDA)

N(MESON)

N(NEUTRAL)

$\mathrm{N}(\mathrm{N})$

N(PION)

N(PI-)

N(PRONG)

$N(S I C M A O)$

$N(S P E C T)$

N(UNSPEC)

N(UNSPEC)
N(UNSPEC $)+$
$C=$ NUCLEON NUCLEON I=1 INITIAL STATE (AND ELASTIC FINAL STATE). DO NOT USE FOR THE SINGLE

PHYSICAL STATE (P P)

$c=$ PRONG PRODUCED BY NON-STRANGE PARTICLE

NON-RESONANT STATE. TO BE FOLLOWED BY NAMES OF
PARTICLES WHICH WERE PRODUCED IN A NON-RESONANT STATE. E.G. NON-RES $\langle P$ PI $+>$

$C=N U C L E O N$ RHO $I=1 / 2$ FINAL STATE

$C=$ GENERAL NUCLEUS. USE ONLY WHEN THE EXACT NUCLEUS OR NUCLEON IS NOT SPECIFIED. E.G., USE FOR TARGET WHEN DETECTOR IS EMULSION.

$C=I=5 / 2, \quad Y=1$ BARYON OF UNSPEC MASS AND CHC

$C=I=5 / 2, Y=1$ BARYON OF UNSPEC MASS
$C=S=0$ BARYON OF UNSPEC MASS, ISOSPIN, FOR USE IN PROP, RVUE, COMP

$C=I$ UNSPECIFIED, $Y=1$ BARYON OF UNSPEC MASS

$C=I$ UNSPECIFIED, $Y=1$ BARYON OF UNSPEC MASS

$C=$ I UNSPEC, MASS UNSPEC, Y=1 BARYON
$C=$ USED FOR MULTIPLICITY DISTRIBUTION OF AP. USE

ONLY WITH DD=MULT

TOR MULTIPLICITY DISTRIBUTION

$C=A$ COLLECTION OF REACTIONS TO DIFFERENT NUMBERS

OF CHARGED HADRONS. USE ONLY WITH DD=MULT
$C=$ USED FOR MUITIPLICITY DISTRIBUTION OF HEAVY IONS

$C=$ GAMMA MULTIPLICITY, USE WITH DD=MULT ONLY

A COLLECTION OF REACTIONS TO DIFFERENT NUMBERS
OF HADRONS. E.G. 2HADRON OF HADRONS, E.G. 2HADRON, 3HADRON, 4HADRON

$=$ USED FOR MULTIPLICITY DISTRIBUTION OF HELIUM NUCLEI IN FINAL STATE. USE ONLY WITH DDEMULT

$C=A$ COLLECTION OF REACTION TO DIFFERENT NUMBERS OF KOS. USE ONLY WITH DD = MULT DI

$C=$ USED FOR MULTIPLICITY DISTRIBUTION OF K-. USE

$C=A$ COLLECTION OF REACTIONS TO DIFFERENT NUMBERS

TESON.

$C=A$ COLLECTION OF REACTIONS TO DIFFERENT NUMBERS

OF NELTRALS. USE ONLY WITH DD=MULT

$C=$ USED FOR MULTIPLICITY DISTRIBUTION OF $\mathrm{N}$. USE

$C=$ PION MULTIFLICITY, USE WITH DD=MULT ONLY

$C=$ USED FOR MUTIPLICITY DISTRIOUTION OF PI-.

$C=A$ COLLECTION OF REACTIONS WITH DIFFERENT NIMBERS OF PRONGS, E.G. OPRONG, ZPRONG, 4PRONG, ETC. .

$C=A$ COLLECTION OF REACTIONS TO DIFFERENT NUMBERS

C

C= $I=1 / 2, Y=1$ BARYN (NOF

$C=I=1 / 2, Y=1$ BARYON OF UNSPEC MASS

$C=I=1 / 2, \quad Y=1$ BARYON OF UNSPEC MAS 


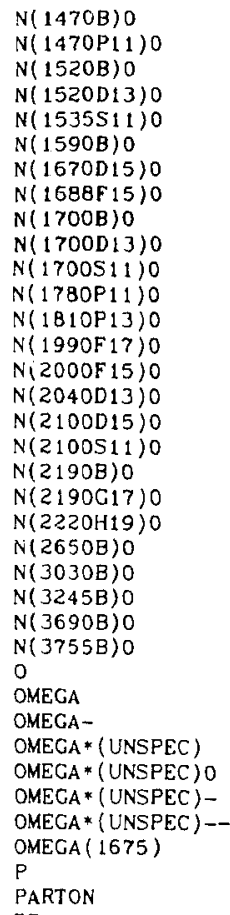

C $(3300-3500) 0$

$\mathrm{PH}$

$\operatorname{PIDEL}(I=1 / 2$

PIDEL $(1=3 / 2)$

PILAM $(I=1) 0$

PILAM $(I=1)+$

$\operatorname{PILAM}(I=1)-$

$\operatorname{PIN}(1=3 / 2)$

PIONS

PIPI $(I=0)$

PIPI $(I=2)$

PIPI $(S 20)_{++}$

$\operatorname{PISIG}([=0)$

$\operatorname{PISIG}(I=0)$

PISIG $(=1)$
PISIG $(I=1) 0$
Appendix VII - Particle Abbrevlations (cont'd)

$N(1470 B)+$

$\mathrm{N}(1520 \mathrm{~B})++$

$\mathrm{N}(1520 \mathrm{D} 13)+$

$\mathrm{N}(1535 \mathrm{~S} 11)+$

$\mathrm{N}(1590 \mathrm{~B})+$

$\mathrm{N}(1670015)+$

$N(1688 F 15)+$

$\mathrm{N}(1700 \mathrm{~B})+$

-1
$N(1700511)+$

$\mathrm{N}(1780 \mathrm{P} 11)+$

$\mathrm{N}(1 \mathrm{~B} 1 \mathrm{OP} 13)+$

$\mathrm{N}(1990 \mathrm{~F} 17)+$

$\mathrm{N}(2000 \mathrm{~F} 15)+$

$\mathrm{N}(2040 \mathrm{D} 13)+$

$\mathrm{N}(2100 \mathrm{D} 15)+$

$\mathrm{N}(2100511)+$

$\mathrm{N}(2190 \mathrm{~B})+$

$\mathrm{N}(2320 \mathrm{H} 19)+$

$\mathrm{N}(2550 \mathrm{~B})+$

$\mathrm{N}(3030 \mathrm{~B})+$

$\mathrm{N}(3245 \mathrm{~B})+$

$\mathrm{N}(3755 \mathrm{~B})+$

C. OXYGEN NUCLEUS

$C=$ MESON RESONANCE

$C=S=-3$ BARYON RESONANCE OF UNSPEC I, MASS

$C=Y=-2$ BARYON OF UNSPEC MASS,

$C=Y=-2$ BARYON OF UNSPEC MASS,

$=Y=-2$ BARYON OF UNSPEC MASS,

$C=$ MESON RESONANCE

$C=$ HYPOTHESTZED PARTICLE

CENERIC NAME FOR EITHER MESON(3500) OR

$=$ PHOSPHORUS -- NOTE NAME IS NOT SAME AS CHEMICAL

SYMBOL

$\cdot C=P I \quad$ DELTA $I=1 / 2$ FINAL STATE

$C=$ PI DELTA I=3/2 FINAL STATE

$C=I=1$ PI LAMBDA SYSTEM OF UNSPEC MASS, FOR PWA

$C=I=1$ PI LAMBDA SYSTEM OF UNSPEC MASS, FOR PWA

$C=I=1$ PI LAMBDA SYSTEM OF UNSPEC MASS, FOR PWA

$C=$ PI NUCLEON $I=1 / 2$ INITIAL STATE (AND ELASTIC

FINAL STATE)

$=$ PI NUCLEON $[=3 / 2$ INITLAL STATE (AND ELASTIC

FINAL STATE). DO NOT USE FOR THE SINGLE

$C=$ TWO OR MORE PIONS

$C=$ PI PI ISOSPIN O STATE

$C=$ PI PI ISOSPIN 1 STATE

PIPI $(S 20)-$ -

$C=1 \approx 0$ PI SIGMA SYSTEM OF UNSPEC MASS, FOR PWA

$\mathrm{C}=1=0$ PI SICMA SYSTEM OF UNSPEC MASS, FOR PWA

$C=I=1$ PI SIGMA SYSTEM OF UNSPEC MASS, FOR PWA
$\operatorname{PISIG}(I=1)+$

PIOS

PIO(S)

PRONG

PSI $(3700) 0$

PSI $(4100) 0$

PT

PHI GH( 1340$)$

OLOW( 1240$)$

QUARK

OUARK $(1 / 3)$
QUARK $(2 / 3)$

$0(1240-1400) 0$

RHOPI (S21)++

RHOPI (S21)--

RHOPRIME $(1250) 0$

BHOO

RHO $(2100) 0$

SHOWER

SIGMAO

SIG(UNSPEC) 0

SIG(UNSPEC)+

SI G(UNSPEC)-
SIG $(1385 P 13) 0$

SIG $(1440 B) 0$

SIG (1440B)

SIC(1440B)-

$\operatorname{SIG}(1480 B) 0$

$\operatorname{SIG}(1480 B)+$

$\operatorname{SIC}(1480 B)-$

$\operatorname{SIG}(1580 D 13) 0$

$\operatorname{SIG}(1620 B) 0$

SIG(1620B)+

SIG(1620s 11$)$

SIG(1660P11)0

$\operatorname{SIG}(1670 B))^{\circ}$

$\operatorname{SIG}(1670 B)+$

$\operatorname{SIG(1670B)-}$

SIG(1670D 13)0

SIG (1690B)O

SIG (1690B)+

SIC(1750S 11$)$

SIG(1765D15)0

SIG (1770P 11$) 0$

$\operatorname{SIG}\left(1840 P_{13}\right) 0$

$\operatorname{SIG}(1915 B) 0$

$\operatorname{SIG}(1915 B)$

$\operatorname{SIC}(1915 B)-$

SIG(1915F15)0
Appendix VII - Particle Atbreviations (cont'd)

$C=I=1$ PI SIGMA SYSTEM OF UNSPEC MASS, FOR PWA

\section{$C=2$ OR MORE PIOS}

$P I-$

$C=$ A CHARGED TRACK ORIGINATING FROM THE PRIMARY

INTERACTIONS. ASSUMES OBSERVED OR UNOBSERVED SPECIFIALLY MENTIONED.

$C=$ PLATINUM NUCLEUS

OHIGH(1340)+ OHICH(1340)

OLOW(1240)+

$C=$ OUARK OF UNSPECIFIED CHARGE

$C=$ QUARK OF CHARGE $1 / 3$
$C=$ QUARK OF CHARGE $2 / 3$

$Q(1240-1400)+\quad Q(1240-1400)-$

$\mathrm{C}=A$ A RHO PI RESONANCE

PHOPRIME (1250)+ RHOPRIME(1250)-

RHOPRIME $(1600)+$ RHOPRIME $(1600)-$

RHO +

RHO(2100)+ RHO(2100)-

$C=$ INTERMEDIATE SCALAR BOSON

$C=$ SHOWER TRACK

$C=I=1, Y=0$ PART ICLE OF UNSPEC MASS

$C=I=1, Y=0$ PART ICLE OF UNSPEC MASS

$\operatorname{SIG}\left(1385 \mathrm{Pi}^{3}\right)+\quad$ PARTICLE OF UNSP

$C=I=1, Y=0$ BUMPS

$C=I=1, Y=0$ BUMPS

$C=I=1, \quad Y=0$ BUMPS

$C=I=1, \quad Y=0 \quad$ BUMPS

$C=I=1, Y=0$ BUMPS

IIG(1580D13)+ SIG(1580D13)-

$C=I=1, Y=0$ BUMPS

$C=I=1, Y=0$ BUMPS

SIG(1620S11)+ SIG(1620S11)-

IG(1660P11)+ SIG(1650P11)-

$C=I=1, \quad Y=0$ BUMPS

$\mathrm{C}=\mathrm{I}=1, \quad Y=0$ BUMPS

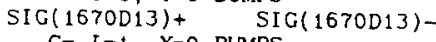

$C=I=1, Y=0$ BUMPS

$C=I=1, Y=0$ BUMPS

SIG(1750S11)+ SIG(1750S11)-

SIG(1755D15)+ SIG(1750S11)-

SIG(1770P11)+ SIG(7770P11)

SIG(1840P13)+ $\quad$ SIG(1770P11)-

SIG(1880P11)+ SIG(1880P11)-

$\begin{array}{lll}C=I=1, & Y=0 & \text { BUMPS } \\ \mathrm{C}=I=1, \quad Y=0 & \text { BUMPS }\end{array}$

$C=I=1, Y=0$
$\operatorname{SIC}(1915 \mathrm{~F} 15)+\quad$ SUMPS
SIG(1915F15)- 
Appendix VII - Partlcle Abbrevlations (cont'd)

SIG(1940D13)0
SIG(2000S11)0
SIG(2030B)0
SIG(2030B)+
SIG(2030B)-
SIG(2030F17)0
SIG(2070F15)0
SIG(2080P13)0
SIG(2100G17)0
SIG(2210H111)0
SIG(2215G19)0
SIG(2250B)0
SIG(250B)+
SIG(2250B)-
SIG(2260D15)0
SIG(2455B)0
SIG(2455B)+
SIG(2455B)-
SIG(2620B)0
SIG(2620B)+
SIG(2620B)-
SIG(3000B)0
SIG(3000B)+
SIG(3000B)-
STRACE
STRANGEO
SU
SO
S+
S-
S*(993)
S(1930)0
T
TA
TACHYN
TH
T(2200)0
U
UNLINKED

UNSPEC

UNSPEC+ UPSI $(5970) 0$ $\mathrm{U}(2360) 0$

VEE

WT

$w_{w+}^{w 0}$

W+
WE
XE
XIO

$\mathrm{XE}$

$\mathrm{XI} * 3 / 2$ (UNSPEC)
$\mathrm{XI} * 3 / 2$ (UNSPEC)

SIG(2260D15)+ SIG(2260D15)-
SIG(1940D13)+ SIG(1940D13)-

G(2000S11)+ SIG(2000511)-

$\begin{array}{llll}C=I=1, & Y=0 & \text { BUMPS } \\ C=I=1, & Y=0 & \text { BUMPS }\end{array}$

$C=1=1, Y=0$ BUMPS

SIG(2030F17)+ SIG(2030F17)-

SIG(2080P13)+ SIG(2070F15)-

SIG $(2100017)+$ SIG $(2100017)-$

SIG(2210H111)+ SIG(2210H111)

$\operatorname{SIG}(2215 \mathrm{C} 19)+\operatorname{SIG}(2215 \mathrm{G} 19)-$

$C=I=1, Y=0$ BUMPS

$C=I=1, \quad Y=0$ BUMPS

$C=I=1, Y=0$ BUMPS

$C=I=1, \quad Y=0$ BUMPS

$C=I=1, \quad Y=0$ BUMPS

$C=I=1, \quad Y=0 \quad$ BUMPS

$C=I=1, \quad Y=0$ BUMPS

$C=I=1, \quad Y=0$ BUMPS

$\begin{array}{lll}C=I=1, & Y=0 & \text { BUMPS } \\ C=I=1, & Y=0 & \text { BUMPS }\end{array}$

$C=$ UNSPECIFIED STRANGE PARTICLE

= NEUTRAL STRANGE PARTICLE $C=$ SAME AS STRANGE

UETECAL SHARCE IS SPECIE CE

SULFUR NUCLEUS -- NOTE NAME IS NOT SAME AS

CHEMICAL SYMBOL

$C=$ INTERMEDIATE SCALAR BOSON

C INTERMEDIATE SCALAR BOSON

$C=$ PTER ODIATE SCALAR BOSON

$5(1930)+\quad S(1930)-$

$C=$ TANTALUM NUCLEUS

$C=$ THORIUM NUCLEUS

$T(2200)+$

$T(2200)$

PSEUDO-PARTICLE USED AS A COMPLETE REACTION BY IDPLIES TOAT DD' S IN SAME RODE APPLY IN TE

$C=$ PARTICLE OF UNSPECIFIED TYPE SEARCH. NORMALLY ITS USE SHOULD BE ACCOMPAN BY A DATA COMAENT DESCRIBING THE PARTICLE OR SEARCH. USE FOR PARTICLES NOT YET FOUND AND OF UNKNOWN PROPERTIES. PRINCIPALLY FOR USE IN PROP $C=$ SAME AS UNSPEC EXCEPT THAT IT HAS CHARGE+
$C=$ SAME AS UNSPEC EXCEPT THAT IT HAS CHARGE-

$(2360)+$

$C=$ NEUTRAL OF UNSPECIF

$C=$ INTERMEDIATE VECTOR BOSON

C $=$ TUNGSTEN NUCLEUS -- NOTE NAME IS NOT SAME AS CHEMICAL SYMBOL

$C=$ INTERMEDIATE VECTOR BOSON

$\mathrm{C}=$ INTERMEDIATE VECTOR BOSON

C= XENON NUCLEUS

$\begin{array}{lll}C=I=3 / 2, & Y=-1 & \text { BARYON OF UNSPEC MASS } \\ \mathrm{C}=[=3 / 2, & Y=-1 & \text { BARYON OF UNSPEC MASS }\end{array}$
Appendix VII - Particle Abbreviations (cont'd)

$\mathrm{XI} * 3 / 2$ (UNSPEC) +

$X I * 3 / 2($ UNSPEC)
$\mathrm{XI} * 3 / 2$ (UNSPEC)

$\mathrm{XI} *(\mathrm{UNSPEC})$

XI * (UNSPEC) 0

$\mathrm{XI} *(\mathrm{UNSPEC})+$

$\mathrm{XI} *$ (UNSPEC)
$\mathrm{XI} *($ UNSPEC $)-$

$X I$ (UNSPEC)

XI (UNSPEC) 0

XI $(1530 P 13) 0$

$\mathrm{XI}(1630) 0$

XI $(1820) 0$

$X I(1940) 0$

XI $(2030) 0$
$X I(2250) 0$

$X I(2250) 0$
$X I(2500) 0$

XI $(2500)$

$X_{1}(1440) 0$

$x 1(1795) 0$
$X(1690) 0$

$x(2500-3600)$

$X(2750) 0$

$Y * Z$ (UNSPEC)
$Y * 2$ (UNSPEC)

$Y * 2$ (UNSPEC) ++
$Y *($ UNSPEC)

$Y *($ UNSPEC) 0

$Y *($ UNSPEC $)+$

Y*(UNSPEC)-

ZO(UNSPEC)

$\mathrm{ZO}(1780)+$

ZO(1865)+

1 (UNSPEC) 0

$Z_{1}($ UNSPEC $)+$

$Z_{1}(1900)++$

$Z_{1}(2150)+0$
$Z_{1}(2150)+$

$Z_{1}(2150)+$
$Z_{1}(2150)++$

$21(2500) 0$

$21(2500)+$

$\mathrm{Z} 1(2500)++$

$\mathrm{Z} *($ UNSPEC)

$Z *($ UNSPEC $) 0$

$Z *($ UNSPEC $)++$

OPRONG

(GAMAMAS)

(HADRONS)

(PIOS)

GE. 4 PRONG

.GT. 10 PRONGS
$C=I=3 / 2, Y=-1$ BARYON OF UNSPEC MASS

$C=I=3 / 2, Y=-1 \quad$ BARYON OF UNSPEC MASS

$C=I=U N S P E C, Y=-1$ BARYON OF UNSPEC MASS

$C=Y=-1$ BARYON OF UNSPEC MASS,

$C=Y=-1$ BARYON OF UNSPEC MASS,

$C=Y=-1$ BARYON OF UNSPEC MASS,

$C=I=1 / 2, \quad Y=-1$ BARYON OF UNSPEC MASS

$C=I=1 / 2, Y=-1$ BARYON OF UNSPEC MASS

$X I(1530 \mathrm{P} 13)-$

$X I(1630)$
$X I(1820)$

$X I(1940)-$

$x=\frac{1}{x I}(2030)-$

XI $(2500)-$

$X 1(1440)+$
$X 1(1795)+$

$x 1(1440)-$

$C=$ ANY MESON BUMP SEEN IN PRODUCTION EXPERIMENTS IN ANY

$=$ J/PSI, ETC.)

$C=I=2, Y=0$ BARYON OF UNSPEC MASS, $C H C$

$C=Y=0$ BARYON OF UNSPEC ISOSPIN

$C=Y=0$ BAYON OF UNSPEC ISOSPIN AND MASS

$C=Y=0$ BARYON OF UNSPEC ISOSPIN AND MASS

$C=Y=0$ BARYON OF UNSPEC ISOSPIN AND MASS

$C=$ EXOTIC $I=0, Y=2$ BARYON OF UNSPEC MASS

$C=$ EXOTIC $I=0, \quad Y=2$ BARYON OF UNSPEC MASS

$C=$ EXOTIC $I=0, Y=2$ BARYON

$C=$ EXOTIC $I=1, Y=2$ BARYON OF UNSPEC MASS

$C=$ EXOTIC I $=1, Y=2$ BAA

$C=$ EXOTIC $I=1, Y=2$ BARYON OF UNSPEC MASS

$\mathrm{C}=$ EXOTIC $\mathrm{I}=1, \quad Y=2$ BARYON

$C=$ EXOTIC $I=1, Y=2$ BARYON

$C=$ EXOTC $I=1, Y=2$ BARYON

$C=$ EXTIC $I=1, \quad Y=2$ BARYON

$C=$ EXOTIC I=1, Y=2 BARYON

$C=$ EXOTIC $I=1, Y=Z$ BARYON

$C=$ EXOTIC I $I=1, Y=2$ BARYON

$C=$ EXOTIC I $=1, Y=2$ BARYON

$C=$ EXOTIC $I=U N S P E C, Y=Z$ BARYON OF UNSPEC MASS

$\mathrm{C}=$ EXOTIC $Y=2$ BARYON OF UNSPEC MASS

$C=$ EXOTIC Y=2 BARYN OF UNSPC MAS

$C=$ USE FOR REACTIONS WITH NO CHARGED PARTICLES ORIGINATING FROM FRIMARY VTX. IGNORES OBSERVED OR UNOBSERVED VEES.

$C=$ ZERO OR MORE CAMMAS

$C=Z E R O$ OR MORE NEUTRAL PARTICLES -- DO NOT USE FOR MA.CE.O

$C=$ GREATER THAN OR EOUAL TO 4 PRONGS

$C=$ MORE THAN 10 PRONGS 